ARTICLE

DOI: $10.1038 / \mathrm{s} 41467-018-05125-4$

\title{
Excitatory synaptic dysfunction cell-autonomously decreases inhibitory inputs and disrupts structural and functional plasticity
}

\author{
Hai-yan He${ }^{1}$, Wanhua Shen ${ }^{2}$, Lijun Zheng ${ }^{2}$, Xia Guo $^{2} \&$ Hollis T. Cline ${ }^{1}{ }^{1}$
}

Functional circuit assembly is thought to require coordinated development of excitation and inhibition, but whether they are co-regulated cell-autonomously remains unclear. We investigate effects of decreased glutamatergic synaptic input on inhibitory synapses by expressing AMPAR subunit, GluA1 and GluA2, C-terminal peptides (GluA1CTP and GluA2CTP) in developing Xenopus tectal neurons. GluACTPs decrease excitatory synaptic inputs and cell-autonomously decreases inhibitory synaptic inputs in excitatory and inhibitory neurons. Visually evoked excitatory and inhibitory currents decrease proportionately, maintaining excitation/inhibition. GluACTPs affect dendrite structure and visual experiencedependent structural plasticity differently in excitatory and inhibitory neurons. Deficits in excitatory and inhibitory synaptic transmission and experience-dependent plasticity manifest in altered visual receptive field properties. Both visual avoidance behavior and learninginduced behavioral plasticity are impaired, suggesting that maintaining excitation/inhibition alone is insufficient to preserve circuit function. We demonstrate that excitatory synaptic dysfunction in individual neurons cell-autonomously decreases inhibitory inputs and disrupts neuronal and circuit plasticity, information processing and learning.

\footnotetext{
${ }^{1}$ The Dorris Neuroscience Center, Department of Neuroscience, The Scripps Research Institute, 10550 North Torrey Pines Road, La Jolla, CA 92037, USA.

${ }^{2}$ Zhejiang Key Laboratory of Organ Development and Regeneration, College of Life and Environmental Sciences, Hangzhou Normal University, Hangzhou, Zhejiang 310036, China. These authors contributed equally: Hai-yan He, Wanhua Shen. Correspondence and requests for materials should be addressed to W.S.(email: shen@idrbio.org) or to H.T.C.(email: cline@scripps.edu)
} 
A ctivity plays a critical role in the refinement and maintenance of functional neural circuits, which are thought to require coordinated development of two principle components: excitatory and inhibitory neurons ${ }^{1}$. Although proportional co-regulation of excitation and inhibition and a constant excitation/inhibition ratio have been widely observed during circuit development ${ }^{1}$, our understanding of how glutamatergic excitatory inputs affect the development of inhibition at synaptic and neuronal levels remains incomplete. Mounting evidence from different brain regions and species suggests that perturbing activity or sensory experience delays development of inhibition and disrupts the maturation and specification of inhibitory neurons and circuits ${ }^{2-7}$, however most of these studies perturbed activity broadly and were unable to resolve cell-autonomous and circuit-based outcomes. Direct evidence that glutamatergic synaptic inputs drive the cell autonomous development of inhibitory input in individual neurons remains elusive ${ }^{8}$.

As the predominant mediator of fast excitatory synaptic transmission, AMPARs provide the initial depolarization that is essential for the activation of NMDARs and subsequent secondary signal transduction and synaptic plasticity mechanisms. Four types of AMPAR subunit (GluA1-4) form different heteroand homo-dimers of AMARs, with GluA1 and GluA2 being the major AMPAR subunits. Regulation of the trafficking of postsynaptic AMPAR underlies activity-dependent plasticity of synaptic strength ${ }^{9-11}$. Regulatory sites within the C-terminal region of GluA1 and GluA2 subunits are required for synaptic trafficking of AMPARs ${ }^{9,12}$. Expression of peptides corresponding to the GluA C-terminal peptides (GluA1CTP or GluA2CTP) impairs AMPAR trafficking, decreases excitatory synaptic transmission, and disrupts experience-dependent synaptic plasticity ${ }^{13-15}$. GluACTPs are therefore effective tools to disrupt AMPAR-mediated excitatory transmission in individual neurons, permitting study of outstanding questions concerning the role of excitatory synaptic inputs in structural and functional development of neurons and circuits.

Here, we express GluA1CTP or GluA2CTP, referred to collectively as GluACTPs, in individual tectal neurons to assess the effects of impaired excitatory synaptic transmission on inhibitory synaptic inputs and the development of structural and functional properties in excitatory and inhibitory neurons in vivo. We show that GluACTP expression proportionally decreases excitatory and inhibitory synaptic inputs, resulting in a constant balance of excitation to inhibition in both inhibitory and excitatory neurons. In vivo time-lapse imaging demonstrates that deficits in excitatory synaptic inputs have distinct effects on dendritic arbor development and experience-dependent structural plasticity in excitatory and inhibitory neurons. GluACTP-mediated decreases in excitatory and inhibitory transmission also manifest in deficits in visual information processing, recorded as impaired spatial and temporal receptive field properties, as well as visuomotor behavior. Finally, GluACTP expression blocks learning-induced behavioral plasticity. Our results demonstrate that excitatory synaptic dysfunction leads to cell-autonomous inhibitory synaptic dysfunction, which then ramifies to impair neuronal and circuit properties and degrade behavioral performance.

\section{Results}

GluACTP expression reduces $E$ and I synaptic transmission. To test whether decreasing glutamatergic synaptic inputs in individual neurons affects GABAergic synaptic transmission, we sparsely transfected tectal neurons with constructs co-expressing GFP and GluA1CTP or GluA2CTP, and recorded mEPSCs and mIPSCs from GFP+ neurons 5-8 days later (Fig. 1a). mEPSC frequency was significantly reduced in both GluA1CTP and
GluA2CTP-expressing neurons, with no significant change in mEPSC amplitudes (Fig. 1b, c). The decrease in mEPSC frequency likely reflects loss of synapses over several days of GluACTP expression. Interestingly, both the frequency and amplitude of mIPSCs were significantly reduced in GluACTPexpressing neurons (Fig. 1d, e), suggesting that excitatory synaptic inputs govern the development of inhibitory synaptic inputs in a cell-autonomous manner. By contrast, disrupting inhibitory synaptic inputs by interfering with $\mathrm{GABA}_{\mathrm{A}} \mathrm{R}$ trafficking does not affect excitatory input onto the same neurons ${ }^{16}$. Paired pulse ratios of excitatory synaptic currents were comparable in neurons expressing GluA1CTP $(n=5)$, GluA2CTP $(n=5)$, and controls $(n=7)$ (Fig. 1f, g), consistent with a deficit in AMPAR trafficking into postsynaptic sites ${ }^{13-15,17}$.

Tectal neurons receive direct excitatory retinal inputs as well as feed forward and feedback inhibitory inputs within tectal circuits $^{11,18,19}$. To determine if the decreased excitatory synaptic transmission affected the $\mathrm{E} / \mathrm{I}$ balance of evoked synaptic responses, we recorded excitatory excitatory compound synaptic currents (eCSCs) and inhibitory compound synaptic currents (iCSCs) from transfected tectal neurons evoked by full-field visual stimulation in intact animals (Fig. 1h). Visually evoked eCSCs and iCSCs recorded from either GluA1CTP- or GluA2CTPexpressing neurons were significantly smaller than controls (Fig. 1i, j), however, the ratio of total integrated charge transfer between iCSCs and eCSCs remained unchanged between GluACTP-expressing and control neurons (Fig. 1k). These data further demonstrate that interfering with GluA1- and GluA2containing AMPAR trafficking not only decreases excitatory synaptic transmission onto the transfected cells, but also induces a proportional decrease in the inhibitory synaptic transmission onto the same neurons.

Inhibition decreases cell-autonomously in $\mathrm{E}$ and I neurons. Excitatory and inhibitory tectal neurons demonstrate different visual experience-dependent structural and functional plasticity ${ }^{20}$. To test whether disrupting excitatory synaptic transmission affects excitatory and inhibitory neurons differentially, we combined immunohistochemical labeling of the excitatory and inhibitory synaptic markers PSD95 and gephyrin, with GABA immunolabeling to examine the density of excitatory and inhibitory postsynaptic puncta in dendrites of GluACTP-expressing neurons.

In the optic tectum, individual tectal neurons express a mixture of AMPARs with different subunit compositions (GluA2-lacking or GluA2-containing). The proportion of GluA2-lacking to GluA2-containing AMPARs varies among individual tectal neurons such that more immature neurons show higher content of calcium permeable GluA2-lacking AMPARs ${ }^{21}$. Both excitatory and inhibitory neurons express GluA1 and GluA2 in the developing optic tectum, as shown by double immunolabeling with GABA and GluA1 or GluA2 antibodies (Fig. 2a, b).

We examined the effects of GluACTP expression on the density of PSD95 and gephyrin puncta in dendrites of sparsely transfected excitatory or inhibitory tectal neurons. Excitatory and inhibitory tectal neurons receive excitatory and inhibitory synaptic inputs (Fig. 2c). Both PSD95 and gephyrin immunolabeling are highly punctate, with higher puncta density in the neuropil than the somatic region (Fig. 2d). GFP+ dendritic segments were identified as inhibitory or excitatory by GABA immunolabeling. In both excitatory and inhibitory neurons, GluA2CTP expression reduced the density of both PSD95 and gephyrin puncta (Fig. 2e-h), indicating a decrease in the number of both excitatory and inhibitory synapses onto transfected neurons. GluA1CTP expression decreased both PSD95 and gephyrin puncta in excitatory neurons but not in inhibitory neurons. 
a

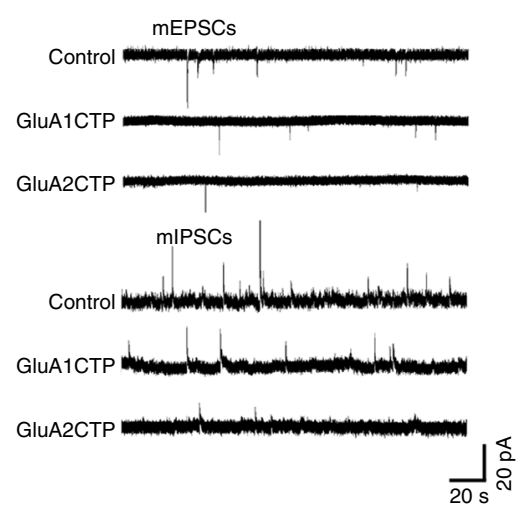

h

Excitatory synaptic currents Inhibitory synaptic currents

Control GluA1CTP GluA2CTP Control GluA1CTP GluA2CTP

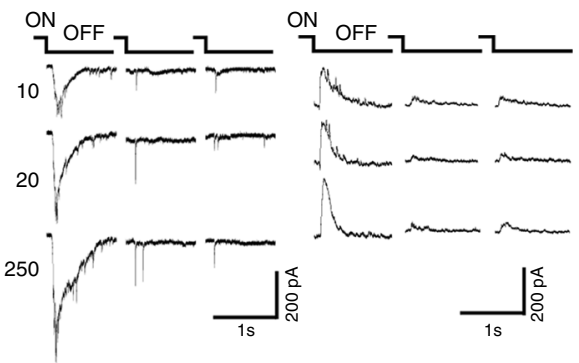

b

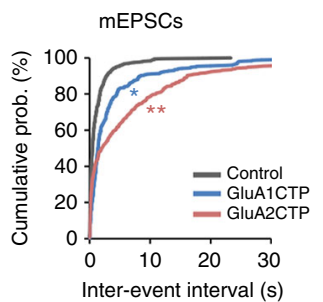

d
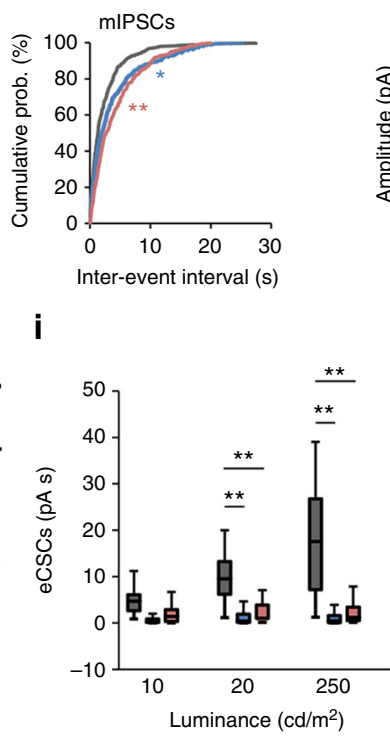

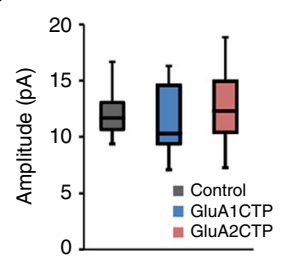

e

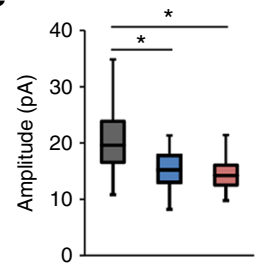

f

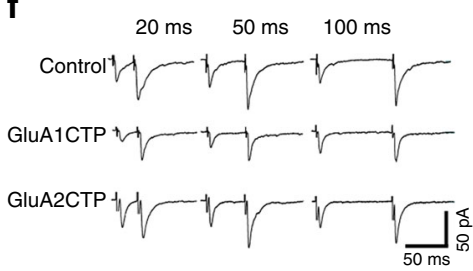

g

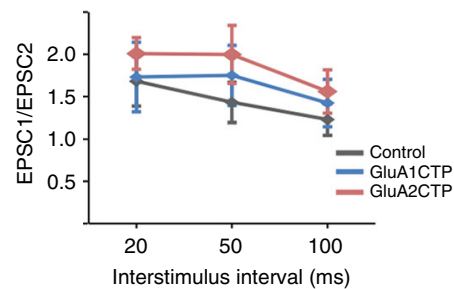

$\mathbf{k}$ j
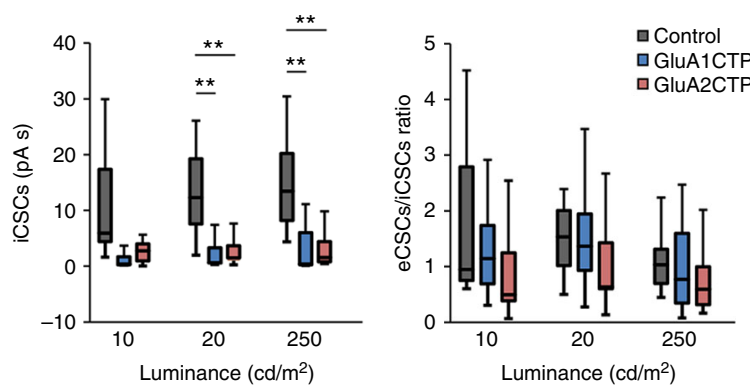

Fig. 1 GluACTP expression reduced both spontaneous and evoked excitatory and inhibitory synaptic transmission in tectal neurons. a Representative traces of mEPSCs and mIPSCs from neurons expressing EGFP only (Control), GluA1CTP, and GluA2CTP, respectively. b Expression of GluA1CTP or GluA2CTP significantly increased inter-event intervals (IEls) of mEPSCs in tectal neurons. ${ }^{\star} P<0.05,{ }^{\star \star} P<0.01$. Kolmogorov-Smirnov test. c Amplitudes of mEPSCs were not significantly affected by GluA1CTP or GluA2CTP expression. Control: $n=14$; GluA1CTP: $n=13$; GluA2CTP: $n=15$. d, e Cumulative distributions (d) and amplitudes (e) of mIPSCs showing GluA1CTP or GluA2CTP expression significantly increased IEl and decreased amplitudes of mIPSCs compared to control neurons. ${ }^{\star} P<0.05,{ }^{\star \star} P<0.01$. Kolmogorov-Smirnov test or ANOVA with Newman-Keuls posthoc test. $\mathbf{f}$ Representative recordings of EPSCs in response to paired stimuli 20,50, and 100 ms apart from neurons in each experimental group. Stimulus artifact was clipped for clarity. $\mathbf{g}$ Paired pulse ratios of EPSC2/EPSC1 were not significantly different between control, GluA1CTP-, and GluA2CTP-expressing neurons. Scale bar: 20 pA, 20 ms. Control: $n=7$; GluA1CTP: $n=5$; GluA2CTP: $n=5$. h Representative traces for visually evoked excitatory CSCs (eCSCs) and inhibitory CSCs (iCSCs) in control, GluA1CTP-, and GluA2CTP-expressing neurons in response to full-field light off visual stimuli at intensities of 10,20 , and $250 \mathrm{~cd} \mathrm{~cm}^{-2}$. i, j Summary data showing that eCSCs (i) and iCSCs (j) in GluA1CTP- and GluA2CTP-expressing neurons are significantly decreased compared to control neurons in response to visual stimuli of 20 and $250 \mathrm{~cd} \mathrm{~cm}^{-2}$ respectively. Control: $n=7$; GluA1CTP: $n=7$; GluA2CTP: $n=7$. $\mathbf{k}$ The ratio of eCSCs to iCSCs in GluA1CTP- and GluA2CTP-expressing neurons remained comparable to control neurons in response to visual stimulation of all luminances tested. ANOVA with Newman-Keuls posthoc test. We record tectal neurons blind to neurotransmitter type. Approximately $70 \%$ of tectal neurons are excitatory neurons, therefore the electrophysiological results most likely reflect changes in the excitatory neurons. For boxplots in this and following figures, the box represents the 25th and 75th percentiles of the samples. The center line represents the median and whiskers depict the full range of observations

Given that over $70 \%$ of tectal neurons are excitatory ${ }^{20}$, these results are consistent with decreased mEPSC and mIPSC frequency seen in electrophysiological recordings from randomly recorded neurons, and demonstrate cell-autonomous loss of inhibitory synaptic inputs induced by decreased excitatory input in both excitatory and inhibitory neurons.

Structural plasticity differently altered in $\mathbf{E}$ and I neurons. To assess effects of disrupted excitatory synaptic transmission on dendritic arbor development and experience-dependent structural plasticity in excitatory and inhibitory neurons, we performed in vivo time-lapse imaging of GluACTP and GFPcoexpressing individual neurons. Excitatory and inhibitory neurons were identified by post hoc GABA immunolabeling (Fig. 3a, b). Total dendritic branch length (TDBL) and total branch tip number (TBTN) from three-dimensional (3D) reconstructions of the imaged neurons indicated that GluA1CTP and GluA2CTP expression in excitatory neurons significantly decreased branch density, without affecting dendritic arbor branching pattern (Fig. 3c-e). GluA2CTP expression also significantly decreased TBTN in excitatory neurons (Fig. 3c). In inhibitory neurons, GluA2CTP expression significantly increased TDBL, without affecting TBTN or branch density (Fig. 3d). Interestingly, GluA2CTP expression also changed the branching pattern of inhibitory neurons, causing neurons to branch significantly farther from the soma (Fig. 3f), possibly reflecting a compensatory response to decreased excitatory inputs, consistent with observations of activity-dependent redistribution of synapse in the absence of normal activity ${ }^{22}$.

Previous studies showed that $4 \mathrm{~h}$ of short term enhanced visual experience (STVE) increased dendritic arbor growth rate in tectal 
a

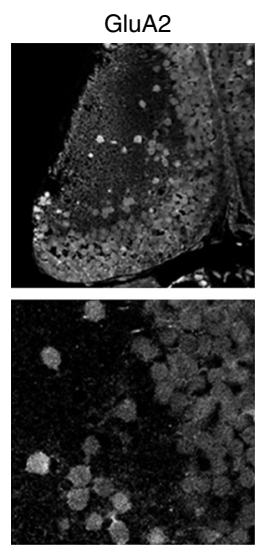

C

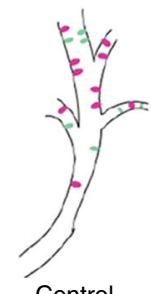

Control

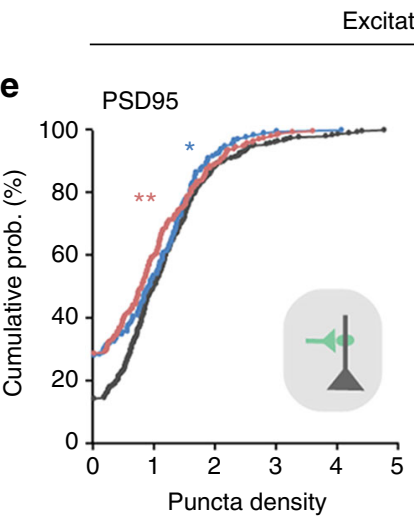

GABA

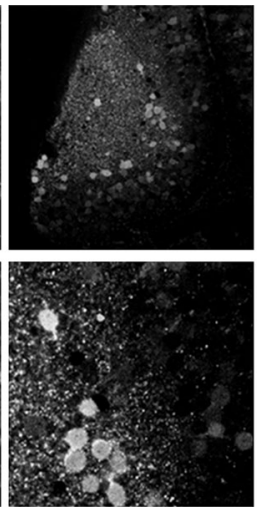

GluA2 GABA DAPI
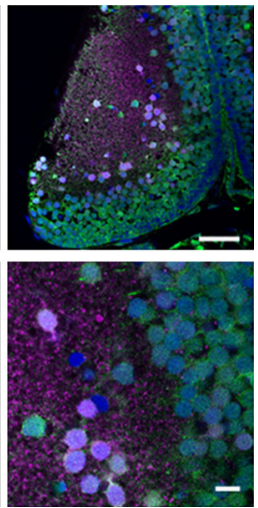

d

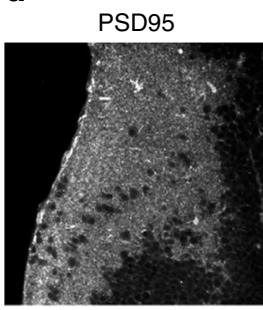

b
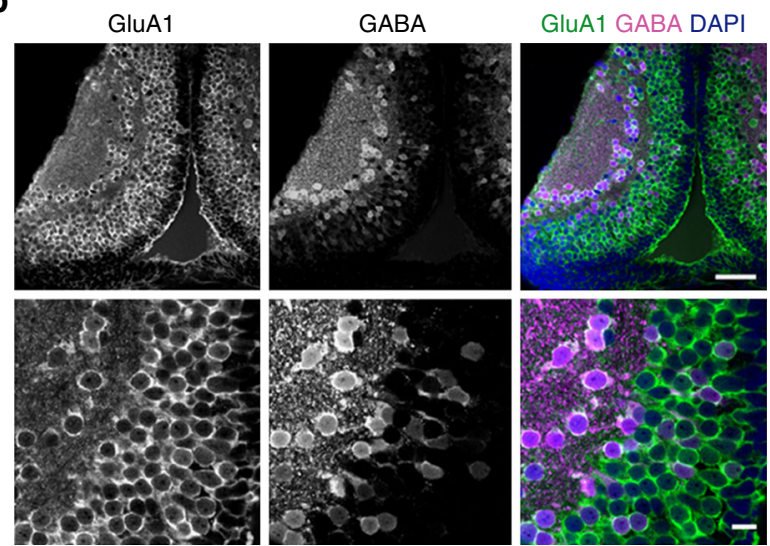

Gephyrin

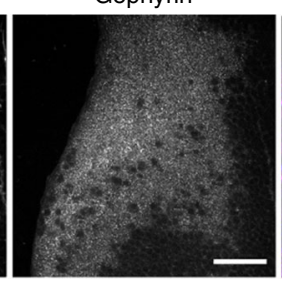

Gephyrin PSD95 GFP

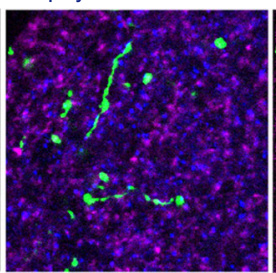

GABA GFP

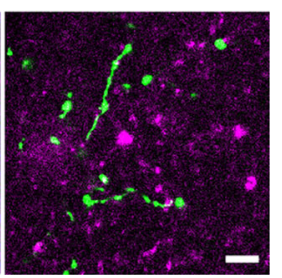

Ecitatory neurons
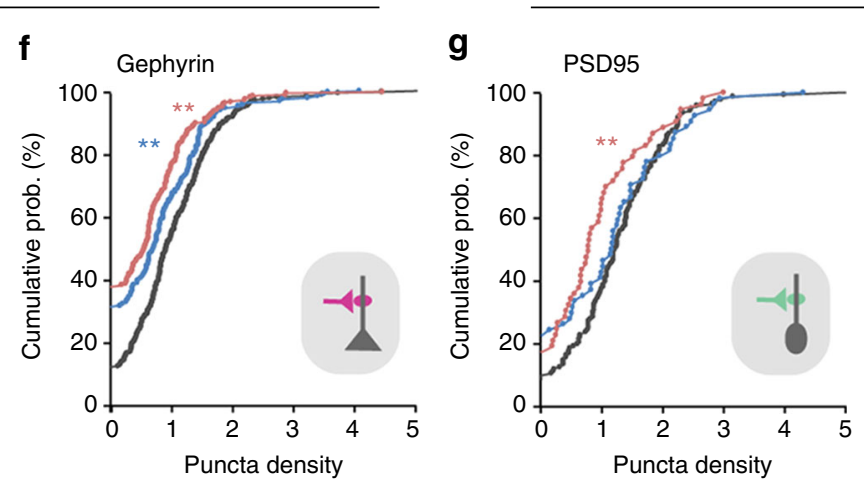

Inhibitory neurons

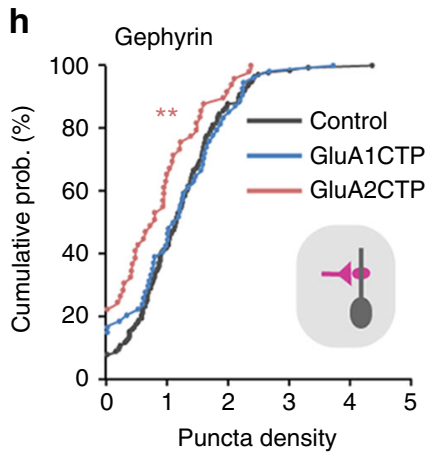

Fig. 2 Decreased excitatory inputs induced cell-autonomous decreases in inhibitory synaptic inputs in both excitatory and inhibitory neurons. a, b Coimmunolabeling of GABA and GluA2 (a) or GluA1 (b) antibodies shows that both GluA1 and GluA2 are widely expressed in the tectum and are found in both excitatory (GABA-negative) and inhibitory (GABA-positive) neurons. Scale bar: top: $50 \mu \mathrm{m}$; bottom: $10 \mu \mathrm{m}$. c Schematic illustrates excitatory and inhibitory synapses on excitatory and inhibitory postsynaptic neurons. d PSD95 and gephyrin immunolabeling in the tectum (left) show high puncta density in the neuropil and relatively low density in the somatic region. Right: representative images of PSD95 and gephyrin puncta in a GFP+ dendritic segment. The identity (excitatory or inhibitory) of the GFP+ dendrite was determined by GABA immunolabeling. Scale bar: left: $50 \mu m$; right: $10 \mu m$. e- $\mathbf{h}$ Summary data showing GluA1CTP and GluA2CTP decreased both PSD95 (e) and gephyrin (f) puncta density in excitatory dendrites (number of dendritic segments: Control: $n=250$; GluA1CTP: $n=170$; GluA2CTP: $n=210$ ). In inhibitory dendrites, GluA2CTP, but not GluA1CTP, significantly decreased PSD95 (g) and gephyrin (h) puncta density (number of dendritic segments: Control: $n=134$; GluA1CTP: $n=54 ;$ GluA2CTP: $n=49$ ). ${ }^{\star} P<0.05$; ${ }^{\star \star} P<0.01 ;$ Kruskal-Wallis test with posthoc Mann-Whitney $U$ test

neurons compared to $4 \mathrm{~h}$ in dark, and that GluACTP expression blocks this visual experience-dependent dendritic arbor structural plasticity $^{14}$. Since these previous studies imaged randomly sampled tectal neurons, the results likely reflect plasticity in excitatory neurons, the majority of tectal neurons. Inhibitory tectal neurons, on the other hand, demonstrate a bimodal experience-dependent plasticity, with an inverse correlation between the valence of plasticity in response to dark and STVE in individual neurons ${ }^{20}$. Furthermore, inhibitory neurons cluster into two functional groups, one group increases dendritic arbor growth rate in response to STVE and decreases arbor growth rate in the dark, while the other decreases arbor growth rate in STVE and increases it in dark.
To test if the bimodal experience-dependent structural plasticity in inhibitory neurons is affected by disrupting excitatory synaptic inputs, we collected time-lapse images of individual tectal neurons coexpressing GFP and GluA1CTP or GluA2CTP in animals exposed to $4 \mathrm{~h}$ of dark followed by $4 \mathrm{~h}$ of STVE (Fig. 4a). In excitatory neurons, GluACTP blocked the STVEinduced dendritic arbor plasticity (Control: $\triangle \mathrm{TDBL}, p<0.01$, $\Delta$ TBTN, $p<0.05$. GluA1CTP: $\Delta$ TDBL, $p=0.52, \Delta$ TBTN,$p=$ 0.83. GluA2CTP: $\Delta$ TDBL, $p=0.94, \Delta$ TBTN, $p=0.82$. Wilcoxon test). Comparing dendritic arbor growth rates over $4 \mathrm{~h}$ in STVE and $4 \mathrm{~h}$ in dark for individual neurons demonstrated that GluACTP selectively blocked the structural plasticity of excitatory neurons in response to STVE but not in dark (Fig. 4b, c). The 
a

Excitatory neurons

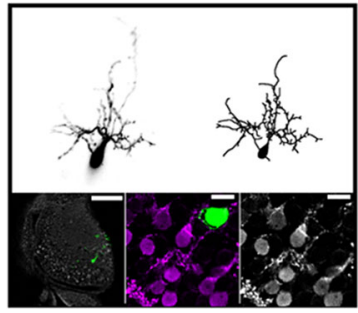

b

Inhibitory neurons

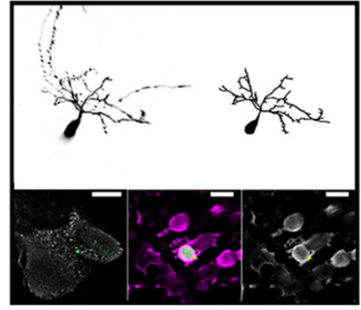

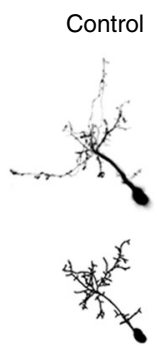

GluA1CTP
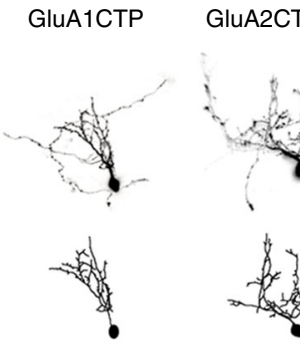

Control

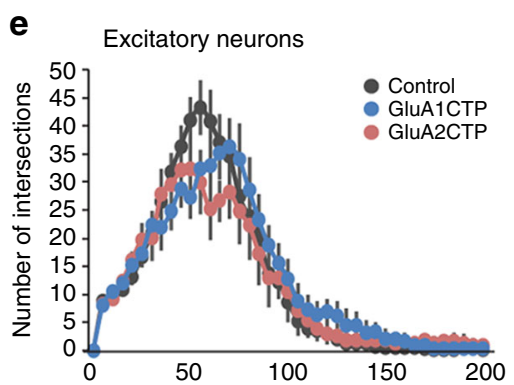

GluA2CTP f

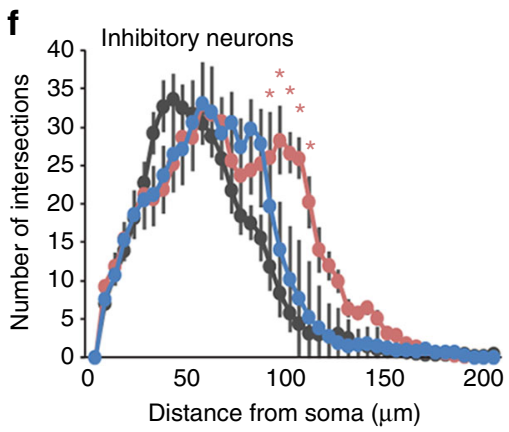

c
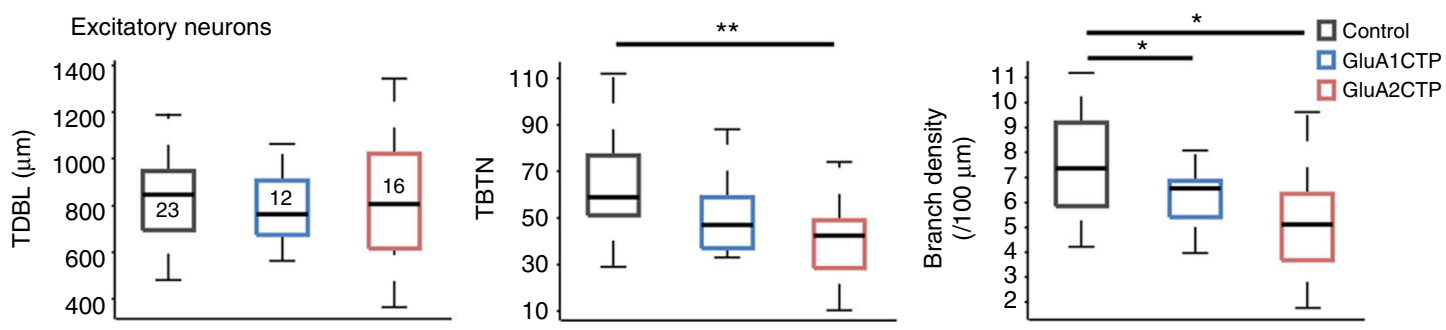

d
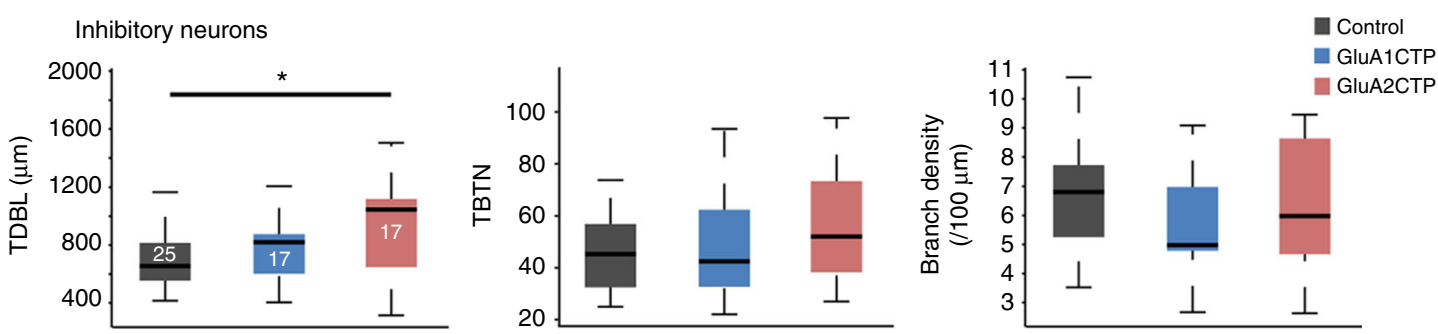

Fig. 3 GluACTPs differentially affect dendritic arbor growth in excitatory and inhibitory neurons. a, b Representative images of excitatory (a) and inhibitory (b) neurons. Left panel: Example of the live image and reconstructed full dendritic arbor of an individual neuron (top). Post hoc GABA immunolabeling (bottom) of the same neuron. Green, GFP; gray/magenta, GABA. Scale bar: left $100 \mu \mathrm{m}$; middle and right $10 \mu \mathrm{m}$. Right panel: Representative live images (top) of inhibitory neurons expressing GFP only (control), GluA1CTP, or GluA2CTP, and reconstructed complete dendritic arbors (bottom). c, $\mathbf{d}$ Summary data of total dendritic branch length (TDBL), total branch tip number (TBTN), and branch density control, GluA2CTP- and GluA1CTP-expressing excitatory (c) and inhibitory (d) neurons. ${ }^{\star} P<0.05$, Kruskal-Wallis test with post hoc Mann-Whitney $U$ test. Number of neurons in each group is shown in the graph. e, f Sholl analysis: neither GluA1CTP nor GluA2CTP affected dendritic arbor branching pattern in excitatory neurons (e). GluA2CTP but not GluA1CTP significantly increased branch density distal to the soma in inhibitory neurons (f) compared to controls. ${ }^{\star} P<0.05,{ }^{\star \star} P<0.01$, Kruskal-Wallis test with post hoc Mann-Whitney $U$ test

pooled population of inhibitory neurons showed no difference in dendritic arbor plasticity between dark and STVE in controls ( $\triangle \mathrm{TDBL}, p=0.38, \Delta \mathrm{TBTN}, p=0.59$ ) or GluACTP-expressing neurons (GluA1CTP: $\triangle$ TDBL, $p=0.06, \quad \Delta$ TBTN, $p=0.48$. GluA2CTP: $\Delta$ TDBL, $p=0.96, \Delta$ TBTN, $p=0.92$, Wilcoxon test). The magnitude of structural responses to either dark or STVE was not different between GluACTP-expressing and control neurons (Fig. 4d). By contrast, plotting dendritic arbor growth rates over $4 \mathrm{~h}$ in STVE versus dark for individual neurons demonstrates that GluACTP expression disrupted the inverse correlation between the valence of structural plasticity in dark and STVE observed in control inhibitory neurons (Fig. 4e).
Application of unsupervised cluster analysis based on $\triangle T B T N$ in response to STVE versus dark clustered control inhibitory neurons into two evenly sized subpopulations, called Group I and Group II neurons (Fig. 5a, Group I: $n=14$; Group II: $n=16$ ). Group I and Group II neurons displayed experience-dependent structural plasticity with opposite valence, accounting for the lack of experience-dependent plasticity in the pooled population (Fig. 4c). The plasticity profile of Group I inhibitory neurons was similar to excitatory neurons, retracting dendrites in the dark and extending them in STVE. The plasticity profile of Group II inhibitory neurons was the opposite, extending dendrites in the dark and retracting them in STVE (Fig. 5a). We applied the same 
a

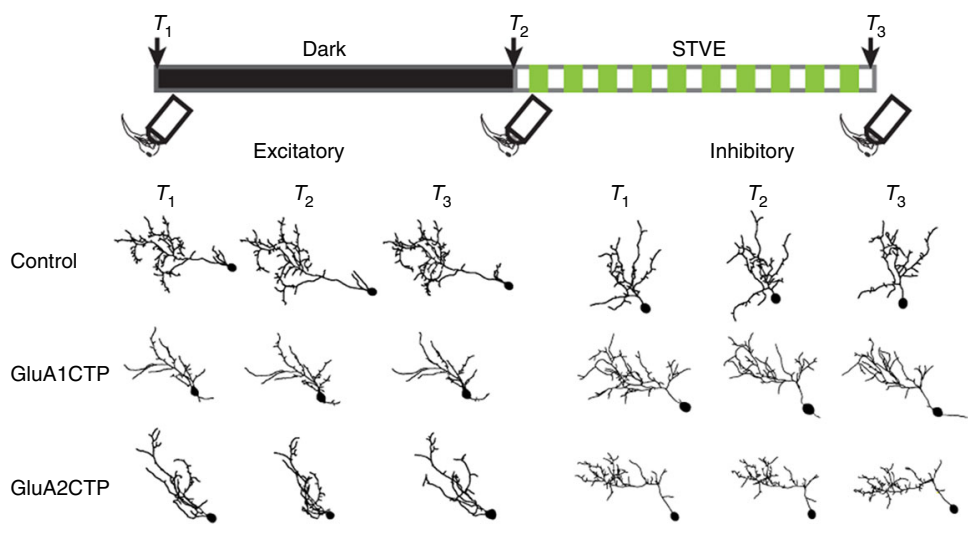

b
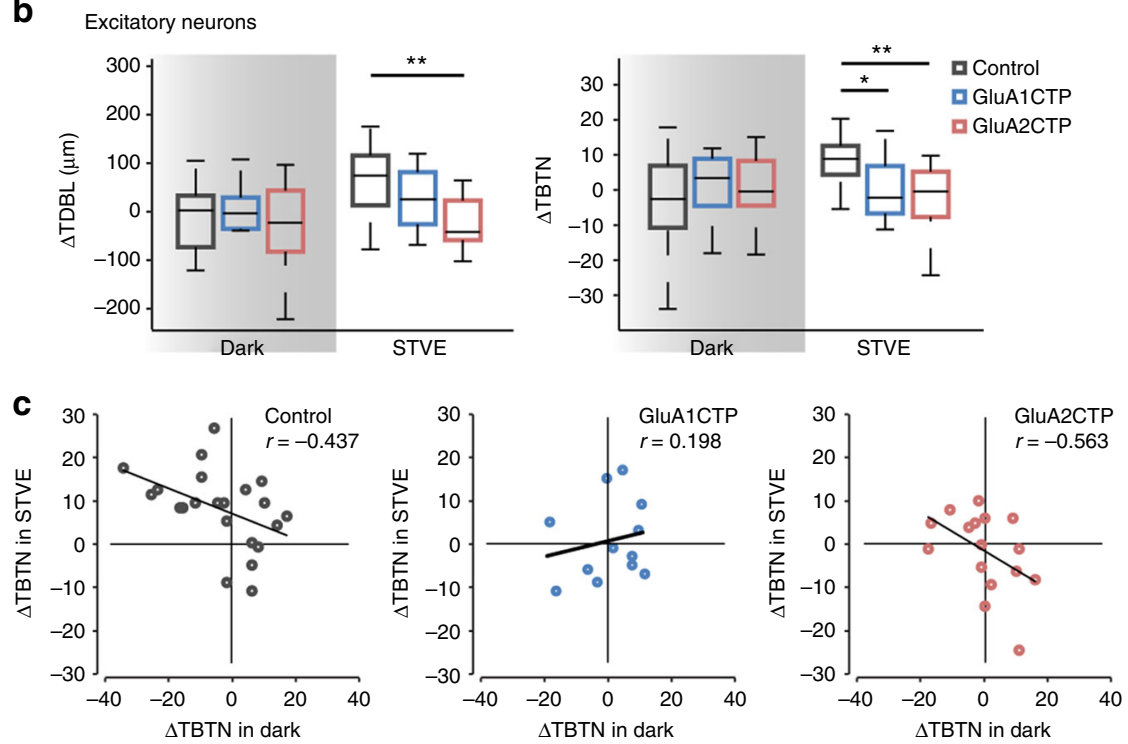

d Inhibitory neurons
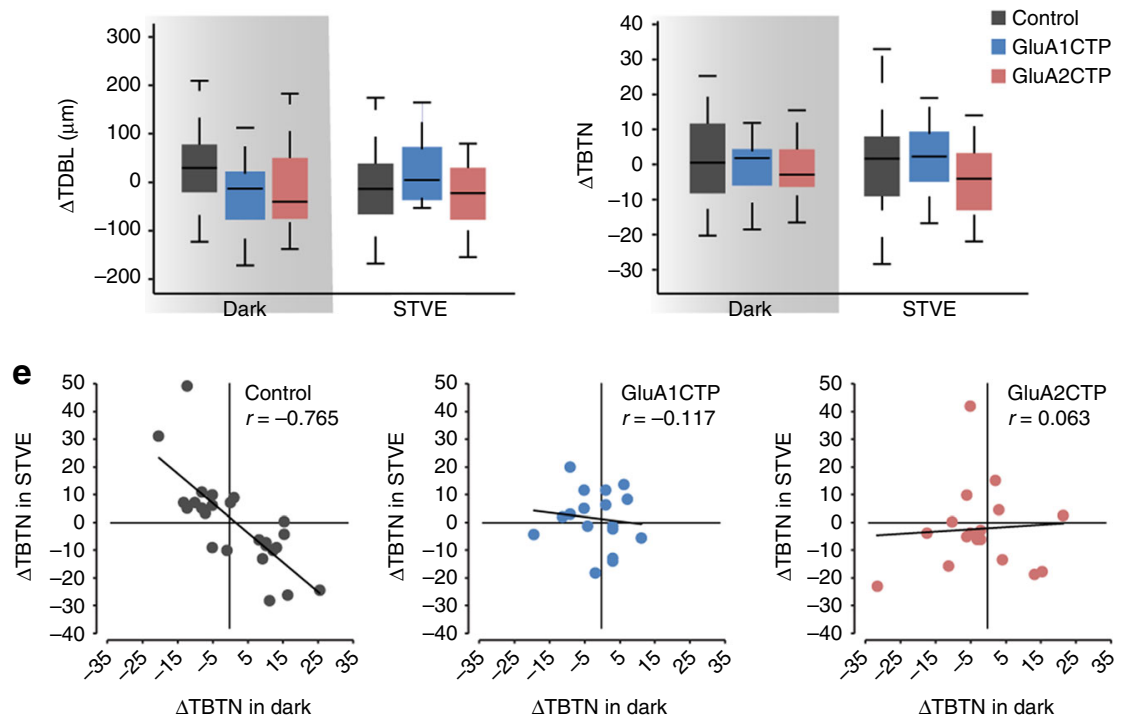

Fig. 4 GluACTP expression disrupts experience-dependent structural plasticity in excitatory and inhibitory neurons. a Representative images of complete dendritic arbor reconstructions from time-lapse images taken before $\left(T_{1}\right)$ and after $\left(T_{2}\right) 4 \mathrm{~h}$ of dark, and after $4 \mathrm{~h}$ of STVE ( $\left.T_{3}\right)$ of individual excitatory and inhibitory neurons in each group. Top: schematic shows the experimental protocol and imaging time course. b Summary data of changes in TDBL ( $\Delta$ TDBL) and TBTN ( $\triangle$ TBTN) during the dark and STVE periods in excitatory neurons. GluA1CTP and GluA2CTP significantly decrease STVE-induced dendritic arbor growth. ${ }^{\star} P<0.05,{ }^{\star \star} P<0.01$, Kruskal-Wallis test with post hoc Mann-Whitney $U$ test. c Scatter plots of $\Delta$ TBTN in response to STVE versus dark in individual excitatory neurons. Rho value of Pearson correlation is shown on each plot. $\mathbf{d}$ Summary data of $\Delta$ TDBL and $\triangle$ TBTN during dark and STVE periods in inhibitory neurons. e Scatter plots of $\triangle T B T N$ in response to STVE versus dark in individual inhibitory neurons 
cluster analysis to GluACTP-expressing inhibitory neurons and assigned the clustered subgroups by the difference between average $\triangle$ TBTN in dark and STVE as in control neurons (Group I: $\triangle$ TBTN in STVE $>\triangle$ TBTN in dark; Group II: $\triangle$ TBTN in STVE $<\triangle$ TBTN in dark). GluA1CTP-expressing inhibitory neurons clustered into two groups with similar structural plasticity profiles as control neurons: Group I neurons retracted dendrites in dark and extended them in STVE. Group II neurons had opposite plasticity profiles. Both groups showed significant differences between plasticity in dark and STVE (Fig. 5b, Group I: $n=11$; Group II: $n=6$ ). By contrast, GluA2CTP-expressing inhibitory neurons clustered into groups that displayed distinct experiencedependent structural plasticity profiles from control neurons: Group I neurons extended dendrites and Group II neurons retracted them in both dark and STVE. Growth rates were not significantly different between dark and STVE in either subgroup (Fig. 5 c, Group I: $n=5, p=0.44$; Group II: $n=12, p=0.41$, Wilcoxon sign rank test), and the experience-dependent bimodal structural plasticity in dark and STVE seen in control inhibitory neurons was abolished. In addition, comparison of the structural plasticity in response to dark or STVE between GluACTPexpressing and control inhibitory neurons showed that within both Group I and Group II neurons, GluA2CTP expression significantly altered the plasticity in response to dark, not STVE (Fig. $5 \mathrm{~d}-\mathrm{g}$ ). By contrast, the plasticity in response to either dark or STVE in GluA1CTP-expressing inhibitory neurons was not different from controls in either group. These results indicate that GluA2 is of particular importance for the bimodal plasticity in inhibitory neurons. Considering that only GluA2CTP expression significantly decreased PSD95 and gephyrin puncta in inhibitory neurons (Fig. $2 \mathrm{~g}, \mathrm{~h}$ ), these results again suggest that, unlike excitatory neurons, inhibitory tectal neurons are more sensitive to the disruption of GluA2-mediated AMPAR trafficking. The loss of the bimodal plasticity response indicates that disrupting excitatory synaptic inputs to inhibitory neurons changed their circuit connectivity ${ }^{20}$.

Using unsupervised cluster analysis, control excitatory neurons cluster into two groups distinguished by their plasticity in the dark (Fig. 6a, Group I: $n=13$; Group II: $n=10$ ): Group I excitatory neuron dendrites retract in dark and extend in STVE $(n=13, p<$ 0.001 , Wilcoxon signed rank test). Group II excitatory neuron dendrites typically grow in both dark and STVE and grow significantly more in dark than in STVE $(n=10, p<0.05)$. GluA1CTP expression significantly changed the plasticity profile in excitatory neurons: Group I neurons retract dendrites in both dark and STVE and Group II neurons extend dendrites in both dark and STVE. Responses to dark and STVE were not different within each group (Fig. 6b. Group I: $n=4, p=0.75$. Group II: $n=$ $8, p=0.46$, Wilcoxon signed rank test). Interestingly, GluA2CTP expression changed the experience-dependent structural plasticity profile of excitatory neurons to a bimodal pattern resembling that of control inhibitory neurons: half retract dendrites in dark and extend them in STVE, and half have the opposite plasticity profile (Fig. 6c, Group I: $n=8$. Group II: $n=8$.). Comparison of the structural plasticity between GluACTP-expressing and control excitatory neurons within each group showed that GluA1CTP and GluA2CTP significantly affected the plasticity in response to STVE but not dark (Fig. 6d-g). The observation that decreased excitatory inputs significantly affected the STVE response in excitatory neurons, and the dark response in inhibitory neurons provides further evidence that the plasticity of inhibitory tectal neurons is actively regulated in dark ${ }^{20,23}$.

Decreased E and I synaptic inputs disrupt RF properties. Interaction of excitatory and inhibitory inputs is thought to be essential for the developmental refinement of visual receptive field (RF) properties ${ }^{1,24,25}$. Here, we showed that interfering with glutamatergic inputs compromises both excitatory and inhibitory inputs yet E/I remains constant. To test whether RF properties are affected by GluACTP expression, we measured the spatial and temporal RF properties in transfected neurons. We recorded spatial RFs in both cell-attached mode, to measure the spiking receptive fields (sRF), and whole-cell mode, to measure excitatory receptive fields (eRF) and inhibitory receptive fields (iRF) respectively in response to light off visual stimuli (Fig. 7a). sRFs, eRFs, and iRFs were all significantly smaller in GluA1CTP- and GluA2CTP-expressing neurons than controls (Fig. 7b-d). GluACTP expression disrupted the convergence of eRF and iRF as shown by significantly greater distance between the eRFs and iRFs centers (Fig. 7e). We generated temporal receptive field (tRF) maps by binning the number of spikes in $100 \mathrm{~ms}$ intervals over the $700 \mathrm{~ms}$ recording period following the OFF stimulus (Fig. 7f). The tRFs in control GFP+ neurons were confined to $200 \mathrm{~ms}$ following the stimulus (Fig. $7 \mathrm{~g}$ ). By contrast, the tRF in GluA1CTP- and GluA2CTP-expressing neurons extended up to $600 \mathrm{~ms}$ after the stimulus. Consequently, the average spike latency and the full width at the half maximal response (FWHM) of the tRFs in GluA1CTP- and GluA2CTP-expressing neurons were significantly greater than controls (Fig. $7 \mathrm{~h}$, i). The decreased convergence of $\mathrm{eRF}$ and $\mathrm{iRF}$ and the increased temporal span of the visually evoked spikes are consistent with a decreased inhibition $^{16,19,24,25}$. Together, these data indicate that decreasing excitatory synaptic inputs and the subsequent cell autonomous decrease in inhibitory synaptic inputs in tectal neurons disrupted the tectal circuits underlying visual information processing and impaired both spatial and temporal RF properties.

Behavioral impairments caused by GluACTP expression. We next addressed whether GluACTP expression affects visual avoidance behavior and behavioral plasticity. The results described above predict two outcomes of GluACTP expression: on one hand, if constant $\mathrm{E} / \mathrm{I}$ is sufficient for circuit function underlying behavior, then the co-regulation of inhibitory inputs in response to GluACTP expression and the resultant constant E/I, predicts that visual avoidance behavior would be intact. By contrast, GluACTP expression impaired visual information processing and RF properties, predicting that circuit function and plasticity would be impaired. We bulk-electroporated tecta with GluA1CTP, GluA2CTP, or GFP expression constructs and evaluated visual avoidance behavior and avoidance behavior plasticity. We first tested whether changes in excitatory and inhibitory synaptic inputs occur after bulk electroporation, which results in $\sim 15-30 \%$ transfection rate ${ }^{16}$. We recorded visually evoked eCSCs and iCSCs from GFP+ neurons expressing either GluA1CTP or GluA2CTP and compared them to control neurons expressing GFP alone. As seen above, expressing GluA1CTP or GluA2CTP more broadly in tectal neurons significantly reduced both the eCSC and iCSC in transfected neurons, so E/I remains comparable to controls (Fig. 8a). The visual avoidance behavior is a tectally mediated behavior in which an animal changes swim trajectory in response to an approaching visual stimulus ${ }^{16}$. Animals improve their avoidance behavior following a visualconditioning training protocol ${ }^{26}$. Control tadpoles avoided dots with diameters ranging from 0.2 to $0.6 \mathrm{~cm}$, with the peak avoidance response to dots of $0.4 \mathrm{~cm}$ diameters (Fig. 8b, c). GluA1CTP- and GluA2CTP-expressing tadpoles had significantly lower avoidance indices to stimuli of $0.4 \mathrm{~cm}$ and $0.2 \mathrm{~cm}$, and GluA2CTP-expressing animals also showed a significantly decreased response to $0.6 \mathrm{~cm}$ dots (Fig. 8c), indicating impaired visual behavior in these animals despite of balanced E/I 

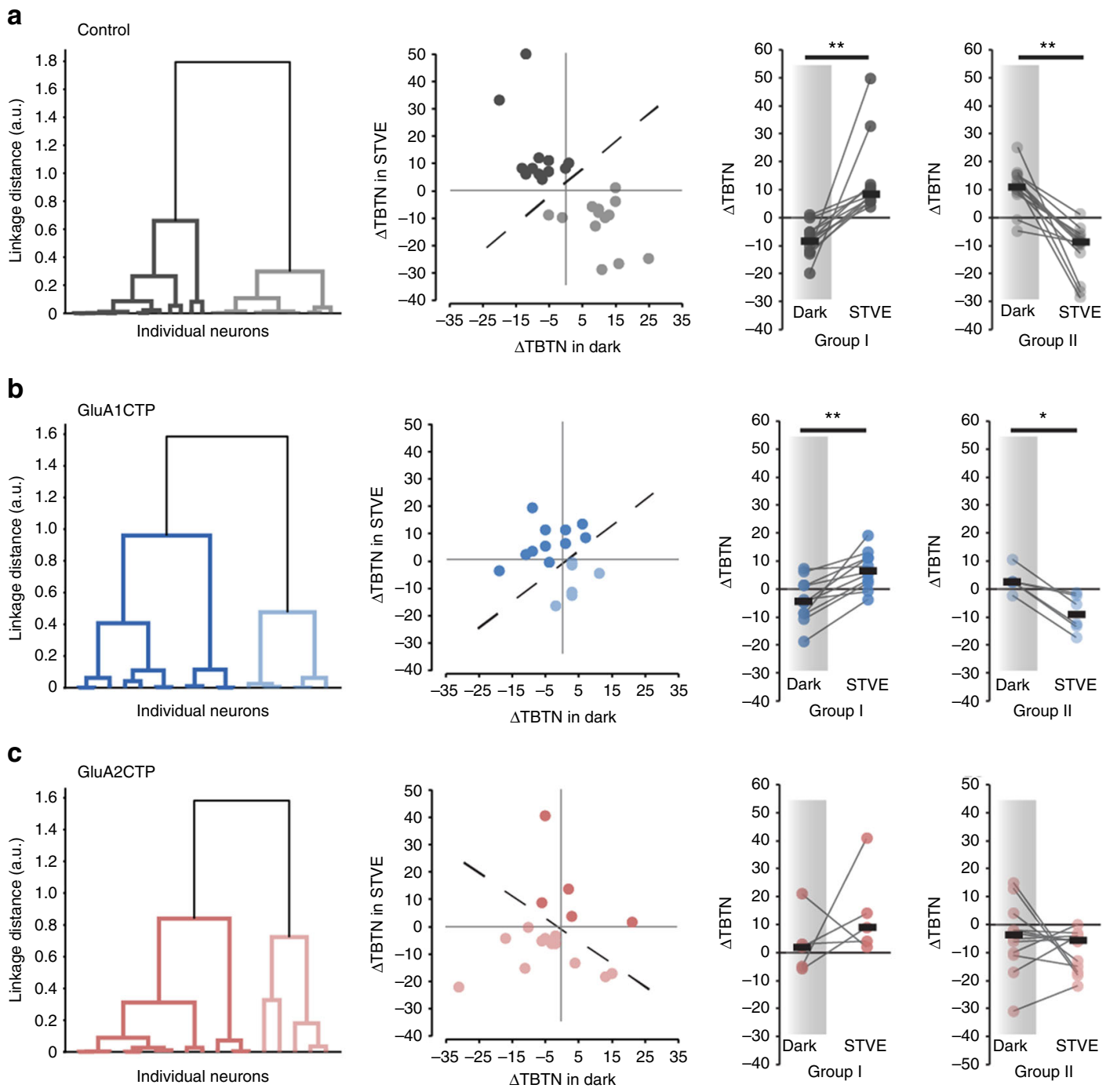

d

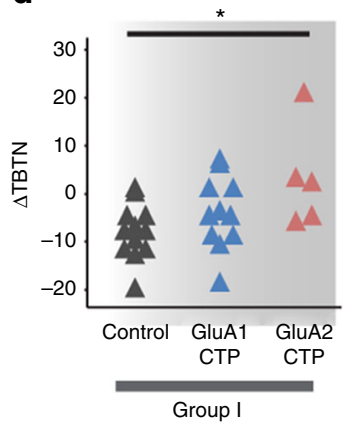

e

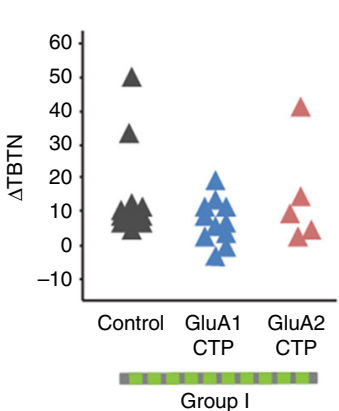

f

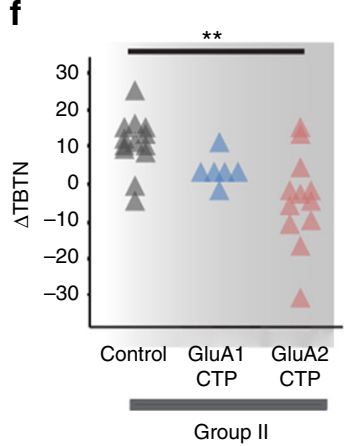

g

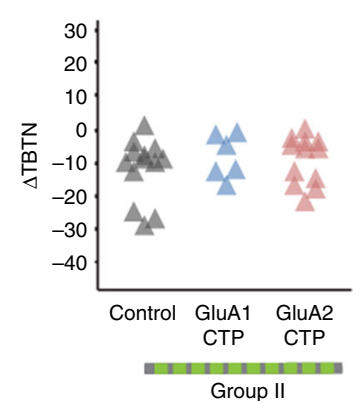

Fig. 5 GluA2CTP expression disrupted the bimodal experience-dependent structural plasticity of inhibitory neurons. a Left: Dendrogram of unsupervised hierarchical cluster analysis of control inhibitory neurons based on changes in TBTN ( $\triangle$ TBTN) in STVE versus dark. Middle: Scatter plots of $\triangle$ TBTN in response to STVE versus dark in individual neurons for Group I and II inhibitory neurons. Right: Summary of $\Delta$ TBTN in dark and STVE for control Group I and II neurons. b Cluster analysis of GluA1CTP-expressing inhibitory neurons. c Cluster analysis of GluA2CTP-expressing inhibitory neurons. ${ }^{\star} P<0.05$, ${ }^{\star \star} P$ $<0.01$, Wilcoxon sign rank test. d, $\mathbf{g}$ Summary data of $\triangle$ TBTN in response to dark $(\mathbf{d}, \mathbf{f})$ and STVE (e, $\mathbf{g})$ in Group I (d, e) and Group II (f, $\mathbf{g})$ inhibitory neurons. ${ }^{\star} P<0.05$, Kruskal-Wallis test with post hoc Mann-Whitney $U$ test

GluA1CTP and GluA2CTP expression also blocked visual conditioning-mediated plasticity of the behavior (Fig. 8d). This learning deficit is consistent with the compromised experiencedependent structural plasticity and visual information processing observed in individual neurons expressing GluACTPs.

\section{Discussion}

Genetic variants of proteins associated with glutamatergic synaptic function, such as CTNAP2 and SHANK3, have been implicated in the etiology of neuropsychiatric disorders, placing excitatory synapse dysfunction in the spotlight as a candidate 
a
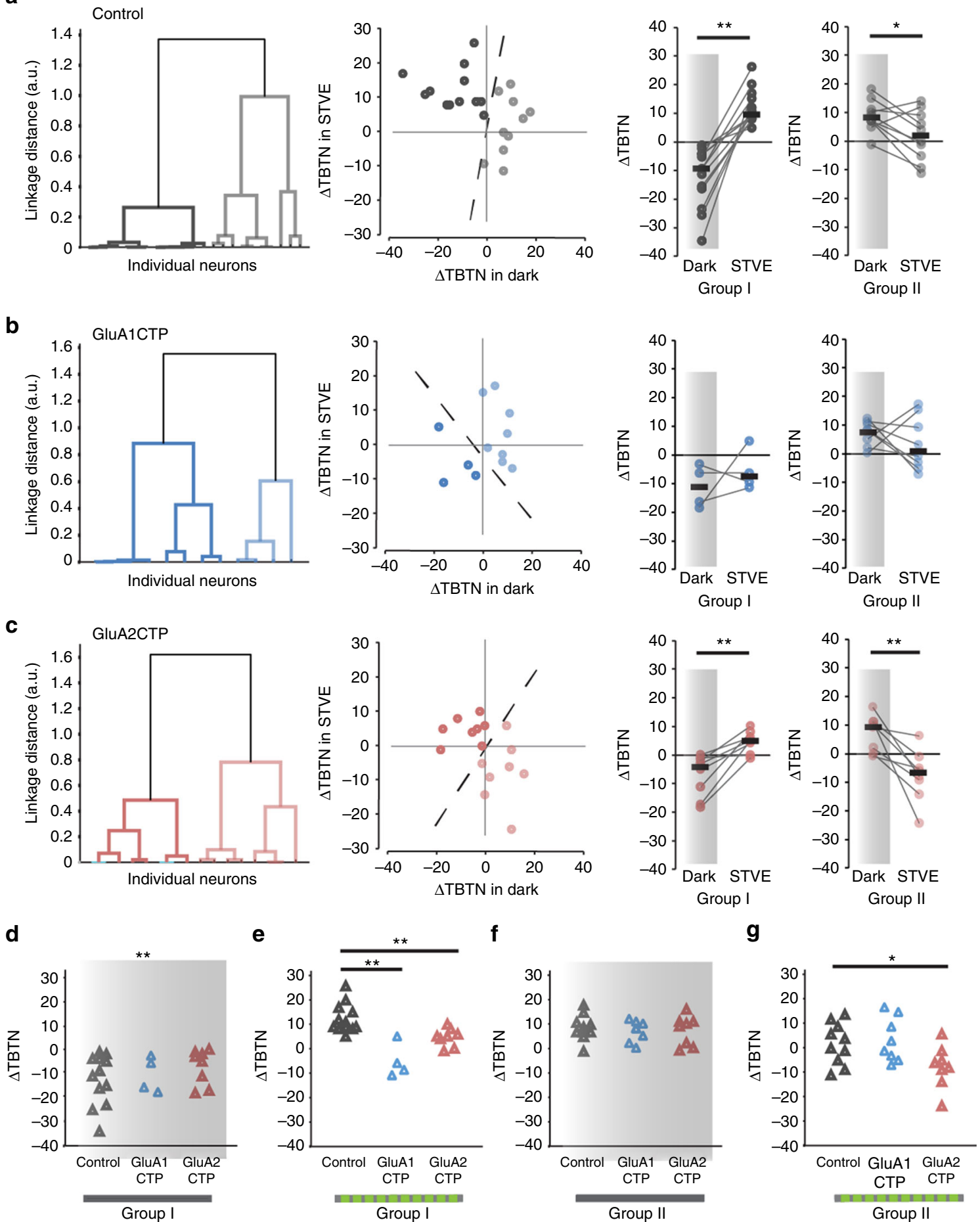

Fig. 6 Cluster analysis of experience-dependent changes in TBTN in individual excitatory neurons. a Left: Dendrogram of unsupervised hierarchical cluster analysis of control excitatory neurons based on $\triangle T B T N$ in STVE versus dark. Middle: Scatter plots of $\triangle T B T N$ in response to STVE versus dark in individual neurons for Group I and II excitatory neurons. Right: Summary of $\triangle T B T N$ in dark and STVE for Group I and II control neurons. b Cluster analysis of GluA1CTP-expressing excitatory neurons. c Cluster analysis of GluA2CTP-expressing excitatory neurons. ${ }^{\star} P<0.05$, ${ }^{\star \star} P<0.01$, Wilcoxon sign rank test. d- $\mathbf{g}$ Summary data of $\triangle$ TBTN in response to dark $(\mathbf{d}, \mathbf{f})$ and STVE $(\mathbf{e}, \mathbf{g})$ in group I $(\mathbf{d}, \mathbf{e})$ and group II $(\mathbf{f}, \mathbf{g})$ excitatory neurons. ${ }^{\star} P<0.05$, Kruskal-Wallis test with post hoc Mann-Whitney $U$ test

mechanism underlying pathogenesis of these disorders ${ }^{27}$. Animal models with these genetic deficits also show reduced inhibitory tone, recapitulating human patient studies $^{28}$. Some of these neurological disorders are thought to have neurodevelopmental origins, such as ASD and schizophrenia, raising the intriguing questions: Is there a causal link between defective excitatory synaptic function during development and reduced inhibition? And how do deficits in excitatory synaptic function lead to deficits in inhibitory function?

Here we examined the role of glutamatergic excitatory synaptic transmission in the development of inhibition and excitation at synaptic, neuronal, circuit and behavioral levels, focusing on 
a

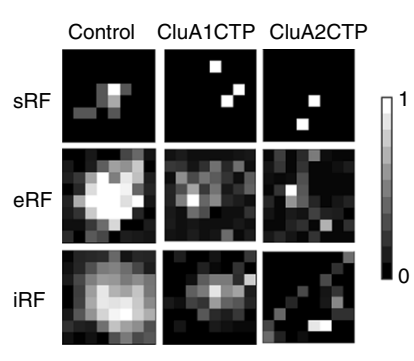

f

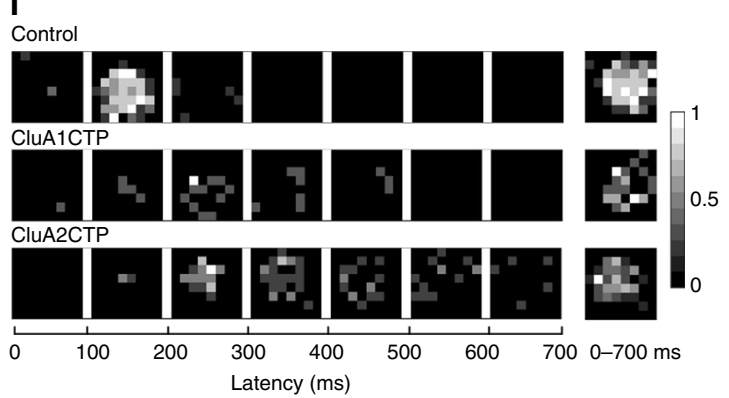

b
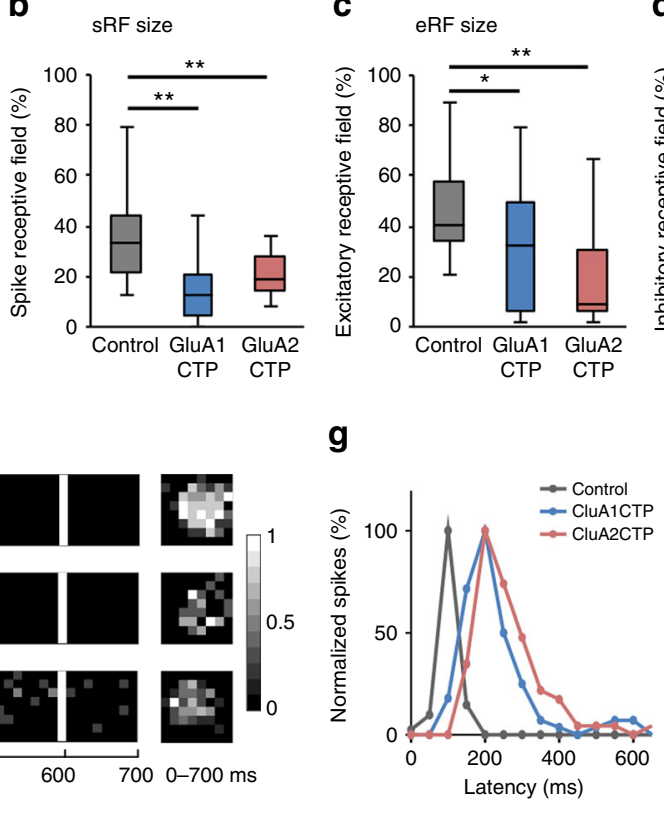

g

d
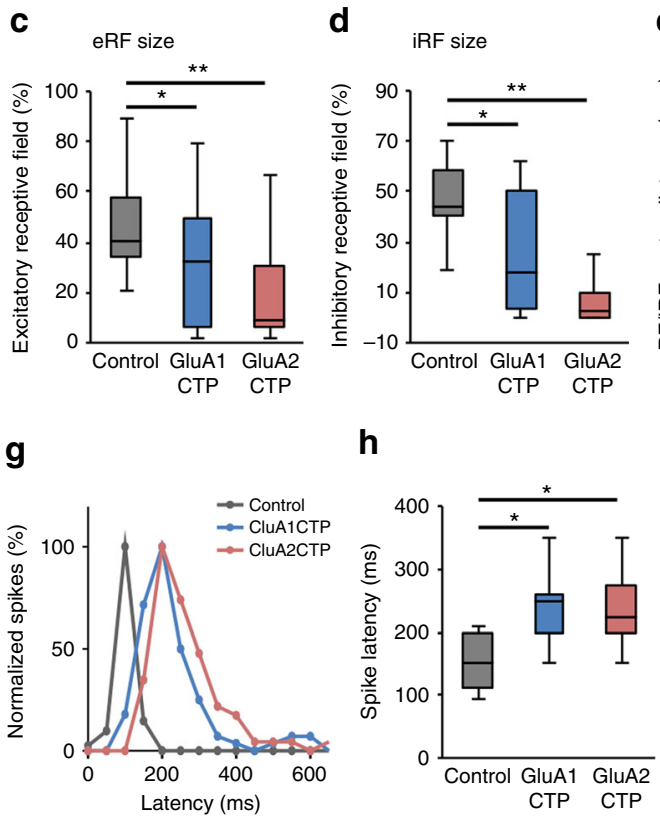

e

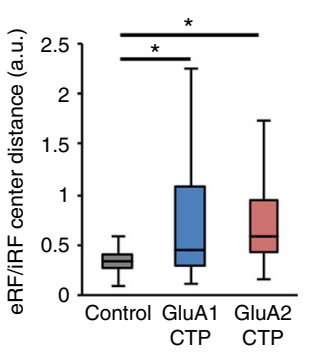

i

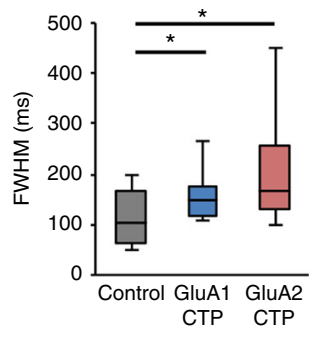

Fig. 7 GluACTP expression disrupts spatial and temporal receptive visual field properties. a Representative maps of spiking RFs (sRFs), excitatory RFs (eRFs), and inhibitory RFs (iRFs) in control, GluA1CTP- and GluA2CTP-expressing neurons. b-d Both GluA1CTP and GluA2CTP expression significantly decreased the size of $\operatorname{sRF}(\mathbf{b}, n=28,14,8)$, eRF $(\mathbf{c}, n=18,19,20)$, and $\operatorname{RRF}(\mathbf{d}, n=18,12,13)$. e The distances between eRF and iRF centers were significantly larger in GluA1CTP- and GluA2CTP-expressing neurons. f Representative tRF maps in control, GluA1CTP- and GluA2CTP-expressing neurons. g Plot of spike numbers over the $700 \mathrm{~ms}$ recording period normalized to peak spike numbers per $100 \mathrm{~ms}$ bin. The control tRF is limited to the first $200 \mathrm{~ms}$ while tRFs in GluA1CTP- and GluA2CTP-expressing neurons were significantly extended and delayed. $\mathbf{h}$, $\mathbf{i}$ Both spike latency (h) and the full width at half maximum (FWHM, i) of the tRF spiking response increased significantly in GluA1CTP- and GluA2CTP-expressing neurons compared to control neurons. Control: $n=12$; GluA1CTP: $n=9$; GluA2CTP: $n=8$. ${ }^{\star} P<0.05$, ${ }^{\star \star} P<0.01$. ANOVA with Newman-Keuls test

inhibitory synapses and neurons. Very little is known about whether and how E/I is maintained in inhibitory neurons. Interfering with AMPAR-mediated inputs specifically in parvalbumin neurons, either with GluA1 or GluA4 knockout, or by manipulating neuronal pentraxins, delayed inhibitory circuit maturation, blocked cortical plasticity, and resulted in behavioral deficits $^{29-31}$. We report a coordinated cell-autonomous reduction in synaptic inhibition in response to decreasing glutamatergic transmission by GluACTP expression in individual neurons. The decreased inhibitory input was not only observed in spontaneous activity but also in visually evoked inhibitory synaptic responses, which drastically altered the spatial and temporal visual receptive field properties in transfected tectal neurons. Disrupting excitatory transmission also blocked experience-dependent structural plasticity in both inhibitory and excitatory neurons. Interestingly, the structural plasticity deficit in inhibitory neurons occurred in the response to dark, whereas in excitatory neurons the deficit occurred in response to visual stimulation. These synaptic and cellular defects translated into behavioral deficits when GluACTPs were expressed more extensively in tectal neurons.

GluACTPs contain core regulatory sites for AMPAR trafficking but lack other functional domains. They compete with endogenous AMPARs for binding partners, thereby interfering with AMPAR trafficking into synapses and decreasing excitatory synaptic transmission. Different mechanisms may underlie the disruption of excitatory synaptic inputs by the expression of GluA1CTP and GluA2CTP due to different synaptic delivery mechanisms of GluA1- and GluA2-containing AMPARs ${ }^{12,13}$. GluA1CTP does not affect basal AMPAR-mediated currents but abolishes activitydependent synaptic potentiation. GluA2CTP significantly decreases basal AMPAR-mediated synaptic transmission, which produces greater synaptic potentiation in response to LTPinducing protocols, but due to impaired synaptic delivery of
GluA2-containing receptors, this increased synaptic strength is not maintained ${ }^{10}$. AMPAR trafficking also affects homeostatic plasticity where GluA2CTP blocks inactivation-induced synaptic scaling of excitatory synaptic inputs ${ }^{32}$. Our data demonstrate celltype specific differences in the effects of GluA1- and GluA2-CTP. While GluA1- and GluA2-CTP affected excitatory neurons similarly, only GluA2-CTP significantly affected inhibitory neurons, both in terms of synaptic inputs, as well as dendritic morphology and structural plasticity. The differential effects of GluA1- and GluA2-CTP in inhibitory neurons are unlikely to result from their differential expression, because both subunits have equally wide expression in all tectal neurons. Inhibitory neurons may employ a CaMK pathway with much slower kinetics than excitatory neurons in coupling excitatory inputs to downstream regulatory pathways ${ }^{33}$. Considering the difference in GluA1 and GluA2 gating kinetics ${ }^{34}$, this may contribute to the different sensitivity of inhibitory neurons to GluA1 expression and GluA2CTP expression. Further investigation will clarify the downstream cell type-specific mechanisms affected by manipulating excitatory input.

Although many studies have examined circuit-wide regulation of $\mathrm{E} / \mathrm{I}$ balance $\mathrm{e}^{1,2,4,35,36}$, whether inhibitory synaptic inputs are regulated cell-autonomously remains unclear ${ }^{37}$. Knocking down all AMPAR-mediated glutamatergic transmission in a small subset of neurons reduced both mIPSC and evoked IPSCs in hippocampal slice culture, suggesting cell-autonomous regulation of $\mathrm{E} / \mathrm{I}^{38}$. Decreasing excitability by potassium channel expression in cortical pyramidal neurons resulted in postsynaptic neuronspecific decrease in inhibitory synaptic inputs from the same presynaptic parvalbumin neurons without affecting excitatory inputs, suggesting cell-autonomous regulation ${ }^{36}$. However, conflicting results had been reported in cultured neurons that similar potassium channel over-expression induced a homeostatic 
a

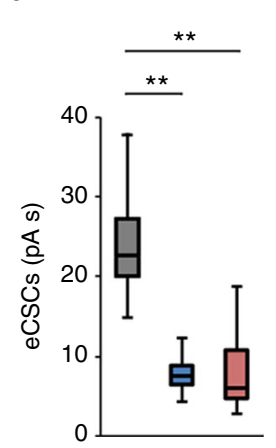

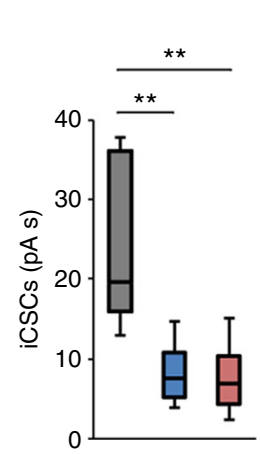

C

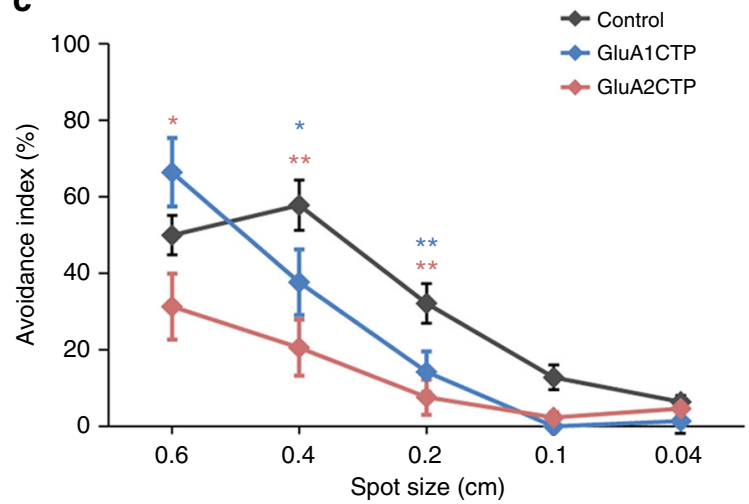

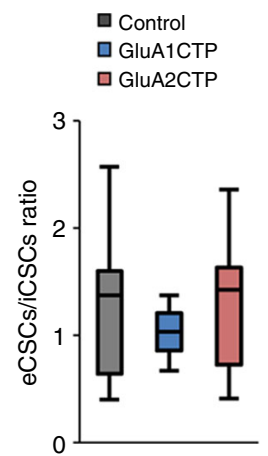

b

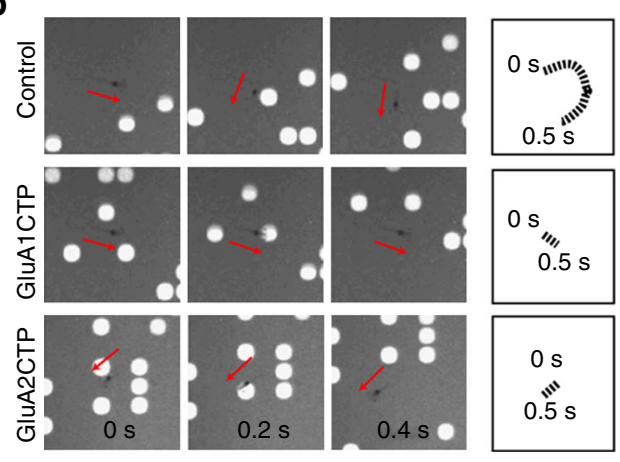

d

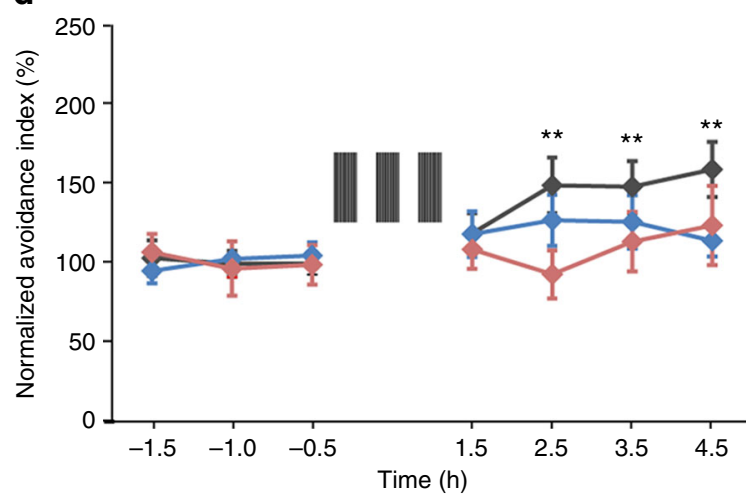

Fig. 8 GluACTP expression impairs visual avoidance behavior and behavioral plasticity. a Expression of GluA1CTP or GluA2CTP with bulk electroporation significantly decreased visually evoked eCSCs and iCSCs in transfected neurons compared to controls. The ratio of eCSCs to iCSCs in GluA1CTP- and GluA2CTP-expressing neurons remained comparable to control neurons. Control: $n=7$; GluA1CTP: $n=8$; GluA2CTP: $n=7$. b Representative snapshots of tadpole behavior in response to upward moving spot stimuli (diameter $0.4 \mathrm{~cm}$ ) in animals expressing GFP, GluA1CTP, or GluA2CTP in tectal neurons. Top panel: Control tadpoles turned to avoid an approaching stimulus. The swim trajectory over $500 \mathrm{~ms}$ is shown on the right. Tadpoles in which the optic tectum was electroporated with GluA1CTP (middle panel) or GluA2CTP (bottom panel) did not change swim trajectories in response to a moving stimulus. c Summary data: avoidance index in response to stimuli of diameters $0.04-0.6 \mathrm{~cm}$ for animals expressing GFP (control, $n=15$ ) or GFP with GluA1CTP ( $n=$ 21) or GluA2CTP $(n=18)$ in the optic tectum. d GluA1CTP $(n=20)$ or GluA2CTP $(n=16)$ expression blocked visual experience-induced enhancement of visual avoidance behavior observed control tadpoles $(n=18) .{ }^{\star} P<0.05,{ }^{\star} \star P<0.01$. ANOVA with Newman-Keuls test. Error bar: mean \pm s.e.m.

increase in excitatory synaptic inputs without changing inhibitory synaptic inputs ${ }^{2,39}$. Our results showing the proportional decrease in excitatory and inhibitory inputs in sparsely transfected GluACTP-expressing neurons provide in vivo evidence that cellautonomous regulation of inhibitory inputs underlies the maintenance of E/I. We previously reported that decreasing inhibition by expressing a peptide interfering with $\mathrm{GABA}_{\mathrm{A}}$ receptor trafficking does not affect glutamatergic synaptic inputs onto the same neuron, thereby disrupting E/I and causing dysfunction of the tectal circuit ${ }^{16}$. These results suggest that cell-autonomous modification of $\mathrm{E} / \mathrm{I}$ is triggered by a direct change in excitatory synaptic input and not by the net change of excitatory inputs relative to inhibitory inputs.

The cell-autonomous downregulation of inhibition following disruption of excitatory inputs may have important implications for the etiology of some neurological disorders. Loss of function of the autism-related cell adhesion molecule CNTNAP2 in cultured cortical neurons caused a cell-autonomous decrease in both excitatory and inhibitory synaptic input ${ }^{40}$. CNTNAP2 preferentially co-localizes with GluA1 and knocking it down in cultured neurons led to abnormal cytoplasmic aggregation of GluA1, suggesting a role in AMPAR trafficking ${ }^{41}$. The dysfunction of inhibitory synaptic transmission observed with CNTNAP2 knockdown may be a secondary consequence of disrupted excitatory transmission due to defective AMPAR trafficking, as we observed here. Another consequence of the coordinated decrease in inhibition induced by decreased excitation is that $\mathrm{E} / \mathrm{I}$ remained relatively stable. E/I is thought to be critical for neural circuit stability and normal brain function ${ }^{1}$. Disrupted E/I is associated with several neurological diseases, including epilepsy, schizophrenia, and autism spectrum disorders ${ }^{42-44}$. Here we showed that even though E/I was resilient to disruption of excitatory inputs, nervous system function was still significantly compromised at both neuronal and circuit levels, shown by abnormal dendritic morphology, altered experience-dependent plasticity, and defective receptive field properties, suggesting that balanced $\mathrm{E} / \mathrm{I}$ is not sufficient to maintain normal brain function. Mice with MECP2 knockout in forebrain excitatory neurons provide another example of simultaneous reduction in excitation and inhibition resulting in functional deficits despite relatively balanced $\mathrm{E} / \mathrm{I}^{45}$. As we showed here, some of the defects in $\mathrm{RF}$ properties resulted from an insufficient excitatory drive, such as the decreased RF size and delayed peak response to visual stimulation. Others are typical results of decreased inhibitory inputs, e.g., decreased temporal precision. Both would contribute to the defective spatial and temporal tuning of visual responses and subsequent impairment of experience-dependent plasticity and animal behavior.

Specific cellular mechanisms underlying the cell-autonomous downregulation of inhibition by decreased excitatory inputs are still unclear. One potential mechanism is retrograde signaling through BDNF, which has been shown to regulate formation and maintenance of inhibitory inputs in response to excitatory inputs $^{46}$. NO has also been proposed as a retrograde messenger 
that mediates heterosynaptic potentiation of GABAergic synap$\mathrm{ses}^{47}$. Previous studies indicate that cell-autonomous regulation of inhibitory synaptic inputs is independent of postsynaptic spiking $^{2}$. Manipulation of neuronal intrinsic excitability with potassium channels expression suppresses neuronal spiking activity but does not prevent postsynaptic depolarization evoked by excitatory synaptic inputs, which allows local $\mathrm{Ca}^{2+}$ influx and can trigger downstream signaling pathways controlling the formation and maintenance of inhibitory synapses ${ }^{48}$. When GABAergic currents are hyperpolarizing and AMPARs are the major source of synaptic depolarization, as is the case in our experiments, disrupting AMPAR-mediated excitatory synaptic inputs reduces the depolarization that is crucial for the activation of NMDARs, which has been shown to be obligatory for the development of inhibitory synaptic transmission ${ }^{49,49}$. Our data provide direct evidence for an essential role for glutamatergic excitatory transmission in the cell autonomous development of GABAergic inhibition and distinguished effects of excitatory inputs on excitatory and inhibitory neurons.

\section{Methods}

Animals and transfection. Albino Xenopus laevis embryos were obtained from either in-house mating or from Xen Express (Brooksville, FL), and reared at 22-23 ${ }^{\circ} \mathrm{C}$ with $12 \mathrm{~h}$ dark $/ 12 \mathrm{~h}$ light cycle in $0.1 \times$ Steinberg's solution (in mM: $58.0 \mathrm{NaCl}$, $0.67 \mathrm{KCl}, 0.34 \mathrm{Ca}\left(\mathrm{NO}_{3}\right)_{2}, 0.83 \mathrm{MgSO}_{4}, 3.0 \mathrm{HEPES}, \mathrm{pH}$ 7.2). Animals were fed from stage 47. All animal protocols were approved by the Institutional Animal Care and Use Committee of the Scripps Research Institute and the local ethics committee of the Hangzhou Normal University. Stage 46-48 animals were anesthetized in $0.02 \%$ MS-222 (Tricane methanesulfonate, Sigma, St. Louis, MO), and the tectum was coelectroporated with pGal4 and either UAS::GluA1CTP-T2A-GFP or UAS:

GluA2CTP-T2A-GFP. Control animals were transfected with pGal4 and UAS::GFP constructs. Animals from the same batches were randomly assigned to each experimental group. All animals were screened 5-8 days post electroporation for proper level of expression before experiments.

Electrophysiology. All recordings were performed at room temperature $\left(20-22^{\circ} \mathrm{C}\right)$. During the recordings, brains were perfused with extracellular saline containing (in mM: $115 \mathrm{NaCl}, 2 \mathrm{KCl}, 3 \mathrm{CaCl}_{2}, 1.5 \mathrm{MgCl}_{2}, 5 \mathrm{HEPES}, 10$ glucose, 0.01 glycine and 0.05 Tubocurarine, $\mathrm{pH} 7.2$, osmolality $255 \mathrm{mOsm}$ ). Visually evoked synaptic currents were recorded from tectal neurons in the middle of the tectum in whole-cell mode using a $\mathrm{K}^{+}$-based pipette solution (in $\mathrm{mM}$ : $110 \mathrm{~K}$-gluconate, $8 \mathrm{KCl}, 5 \mathrm{NaCl}$, 1.5 $\mathrm{MgCl}_{2}$, 20 HEPES, 0.5 EGTA, 2 ATP, and 0.3 GTP). Action potentials were recorded in cell-attached mode. Recording micropipettes were pulled from borosilicate glass capillaries and had resistances in the range of 7-9 M $\Omega$. Liquid junction potential was adjusted during recording. Whole-cell recordings were accepted for analysis from cells in which the series resistance did not change over $10 \%$ and input resistance $(0.7-2 \mathrm{G} \Omega)$ remained relatively constant. Signals were filtered at $2 \mathrm{kHz}$ with a Multiclamp 700A amplifier (Molecular Devices, Palo Alto, $\mathrm{CA}$ ). Data were sampled at $10 \mathrm{kHz}$ using ClampFit 10 (Molecular Devices). Responses, including spikes, eCSCs, and iCSCs to light off stimuli were analyzed by Matlab (The MathWorks, Psychophysics Toolbox extensions).

For whole-cell recordings, tadpoles were anesthetized and tectal lobes were cut along the dorsal midline with a sharp needle. Live tadpoles were immobilized on a sylgard cushion in the recording chamber with one eye facing the center of a backprojection screen. Full-field visual stimuli were generated in Matlab with Physics Toolbox and presented from lowest to highest luminance $\left(10,20\right.$, and $250 \mathrm{~cd} \mathrm{~m}^{-2}$ ) from a projector (Samsung, sp-p310ME LED projector) to the back-projection screen. Each stimulus was presented ten times (Frequency: $0.1 \mathrm{~Hz}$, interval: 0.05 $\mathrm{Hz}$ ). For recording of visually evoked synaptic compound currents in bulkelectroporated animals, tadpoles were transfected with DNA constructs expressing GFP, or GFP with GluR1CTP or GluR2CTP respectively. Animals were screened to select those with dense GFP expression for recordings.

For receptive field mapping, white squares on a dark background were presented in an $8 \times 8$ grid of $0.5 \times 0.5 \mathrm{~cm}^{2}$ non-overlapping squares covering a $4 \times$ $4 \mathrm{~cm}^{2}$ area in the visual field. The entire visual field was mapped by randomly presenting white squares for $1.5 \mathrm{~s}$ with $5 \mathrm{~s}$ intervals. For spike-receptive field mapping, using cell-attached recordings, the response within $700 \mathrm{~ms}$ after the onset of the off stimulus at each grid position was determined as the average number of total spikes per stimulus from two or three repeats. After cell-attached recording, whole-cell voltage clamp recording was accomplished by applying negative pressure. The same visual stimuli were used to measure inhibitory spatial receptive fields and excitatory spatial receptive fields, by holding membrane potential at $0 \mathrm{mV}$ and $-60 \mathrm{mV}$ respectively. Total synaptic charge transfer over $700 \mathrm{~ms}$ from the onset of stimulus was normalized to the peak response and computed by Matlab to show spatial receptive field size. All values larger than three times the standard deviation of spontaneous activity were included in the measurements of spatial receptive fields. The center of the spatial receptive field was defined as the center of the square that elicited the highest responses (maximum number of spikes)

Time-lapse imaging of dendritic arbor and data analysis. Animals were electroporated with DNA constructs at stage of 45-46 and screened for those with sparsely transfected and well-isolated cells. For imaging, animals were anesthetized with $0.01 \%$ MS-222 (Sigma) and were placed in a Sylgard chamber covered by a glass coverslip. Images were collected every $4 \mathrm{~h}$ before and after each visual experience session (dark or STVE). Two-photon $z$-series were collected at $1 \mu \mathrm{m}$ steps with a $20 \times$ water immersion objective (Olympus XLUMPlanFL 0.95NA) at $3-4 \times$ scan zoom using a custom-built microscope modified from an Olympus FV300 system $^{20}$

Complete dendritic arbors of each neuron were reconstructed using a semimanual function in the Filament module of Imaris (Bitplane, US). Total dendritic length and TBTN were automatically calculated by the software. 3D Sholl analysis calculated the number of branches that intersect concentric circles at increasing distances from the cell soma, using a customized Matlab program with reconstructed filament data exported from Imaris. The dendritic structural data of the control group (Figs. 3-6) was a subset of a previously reported data set[20]. These neurons were collected and processed in parallel with the GluACTPexpressing experimental groups.

Immunohistochemistry and image data analysis. For post hoc analysis of neurons imaged for in vivo time-lapse experiments, animals were fixed at the end of the imaging experiment with freshly made $4 \%$ paraformaldehyde and $2 \%$ glutaraldehyde (Electron Microscopy Sciences, Fort Washington, PA) in $1 \times \mathrm{PBS}(\mathrm{pH}$ 7.4) using a Pelco BioWave Pro microwave (Model 36500, Ted Pella, Redding, CA. $350 \mathrm{mV}$ on $20 \mathrm{~s}$, off $20 \mathrm{~s}$, on $20 \mathrm{~s}$, followed by $150 \mathrm{mV}$ on $1 \mathrm{~min}$, off $1 \mathrm{~min}$, on $1 \mathrm{~min})$. The animals were then post fixed at $4{ }^{\circ} \mathrm{C}$ overnight and washed in $1 \times \mathrm{PBS}$ using the microwave ( $150 \mathrm{mV}$ on-off-on, $1 \mathrm{~min}$ each). In total, $30 \mu \mathrm{m}$ vibratome sections of the dissected brains were cut for free-floating immunofluorescence labeling. Sections were incubated in 1\% sodium borohydride (Sigma) in $1 \times$ PBS for 15 min to quench autofluorescence, blocked in $10 \%$ normal goat serum (Jackson $\mathrm{Lab}, \mathrm{ME}$ ) in PBS with $2 \%$ Triton X-100 (PBST) for $1 \mathrm{~h}$ in room temperature, followed by incubation in rabbit anti GABA polyclonal antibody (Sigma A2052, RRID: AB_477652, 1:2000 in PBST with $1 \%$ normal goat serum) for $48 \mathrm{~h}$ at $4{ }^{\circ} \mathrm{C}$. Secondary antibody (goat anti rabbit Alexa Fluor 633, Thermo Fisher Scientific, Cat\# A-21070, RRID: AB_2535731) was diluted 1:500 in PBST and incubated for an hour at room temperature. After $3 \times 15$ min rinses with PBS, sections were mounted on slides in Vectashield Mounting Medium with DAPI (Vector Laboratories, Burlingame, CA). For immunolabeling with GluA antibodies, stage 47 animals were fixed in $4 \%$ PFA and $0.5 \%$ glutaraldehyde. Primary antibodies used include mouse anti GluA2 (N) antibody (1:200, Millipore, Cat\# MAB397, RRID: AB_2113875) and mouse anti GluA1-N antibody (1:500, generous gifts from Dr. R. Huganir, Johns Hopkins University Medical School). For PSD95 and gephyrin puncta analysis, 7 days after electroporation of the corresponding DNA constructs, animals were fixed with $4 \%$ PFA with $1 \mathrm{~h}$ post fixation at room temperature. Free-floating vibratome sections were quenched and blocked as mentioned above, followed by incubation in the primary antibodies including rabbit-anti-PSD95 polyclonal antibody (1:50, Thermo Fisher Scientific, Cat\# 51-6900, RRID: AB_2533914), mouse-anti-gephyrin monoclonal antibody (1:50, Synaptic Systems, Cat\# 147021, RRID: AB_2232546), guinea pig-anti-GABA polyclonal antibody (1:1500, Millipore, Cat\# AB175, RRID AB_91011). Secondary antibodies used include goat-anti-guinea pig Alexa Fluor 568 (Thermo Fisher Scientific, Cat\# A-11075, RRID: AB_141954), goat-anti-rabbit BV421 (BD Biosciences Pharmingen, Cat\# 565014, RRID: AB_2716308), and donkey-anti-mouse Alexa Fluor 647 (Thermo Fisher Scientific Cat\# A-31571, RRID: $\left.A B \_162542\right)$. Brains from control and experimental groups were embedded in the same blocks and processed under exactly same conditions throughout the experiments.

Images of immunolabeled sections for post hoc identification of GABAergic neurons for the in vivo time-lapse imaging experiments were collected on an Olympus Fluoview 500 confocal microscope or a Nikon C2 confocal microscope. GFP fluorescence signal was well preserved in the fixed tissue, thus no antibody was needed for visualization. The GFP+ imaged neurons were located using a $20 \times$ air objective and confirmed by location and dendritic arbor branching patterns. Higher magnification images were then taken at $>3$ different $z$-depth through the soma to examine GABA immunoreactivity. Samples with poor GABA immunostaining were not included in the analysis.

Images of PSD95 and Gephyrin immunolabeling were acquired on a Nikon C2 confocal microscope with a $40 \times$ PlanFluor Oil objective (N.A. 1.3) at $2048 \times 2048$ to achieve a final resolution of $0.15 \mu \mathrm{m}$ pixel $^{-1}$. Analysis of dendritic puncta density was performed using SynPAnal program ${ }^{50}$. Transfected dendritic segments of control or experimental groups were defined solely based on the GFP signal. The dendritic segment was deemed inhibitory if the average GABA immunolabeling intensity within the segment was above a threshold set by mean +2 SD of GABA immunolabeling intensity of three randomly drawn GFP-segments of similar length and width in the neuropil region within the same section adjacent to the 
transfected GFP+ dendritic segment. In a subset of dendritic segments $(n=496)$, for which the soma could be identified, $82.5 \%$ showed GABA immunoreactivity consistent with their soma, confirming the reliability of identifying GABAergic inhibitory dendritic segments based on GABA immunoreactivity within the segments. Puncta density values (per unit length of dendrite) of PSD95 and Gephyrin puncta within the transfected dendritic segments were automatically taken by the program and were normalized to the average puncta density of the neuropil regions within the same section to control for immunostaining variability across sections. All image analysis was done blind to experimental conditions.

Visual avoidance assay and visual conditioning. The visual avoidance assay was conducted as previously described ${ }^{16}$. Tadpoles were placed in an $8 \times 3 \mathrm{~cm}$ tank filled to a depth of $\sim 1 \mathrm{~cm}$ with Steinberg's rearing solution. Visual stimuli were presented to a back-projection screen on the bottom of the chamber using a microprojector ( $3 \mathrm{M}$, MPro110). Videos of tadpoles illuminated by IR LEDs were recorded with a Hamamatsu ORCA-ER digital camera. Visual stimuli were generated and presented by MATLAB. Randomly positioned moving spots of $0.04,0.2$, 0.4 , and $0.6 \mathrm{~cm}$ diameter were presented in random order for $60 \mathrm{~s}$. Visual avoidance behavior was scored as a change in the swimming trajectory in the first ten encounters between each tadpole and moving spots (the percentage of avoidance responses out of 10 encounters, plotted as avoidance index). For visual conditioning, animals were exposed to moving bars $(1 \mathrm{~cm}$ width; $0.3 \mathrm{~Hz}$; Luminance: $25 \mathrm{~cd} \mathrm{~m}^{-2}$ ) continuously for 2 or $4 \mathrm{~h}$, or for three repeats of $5 \mathrm{~min}$ of moving bars interleaved by 5 -min blank, for a total of $30 \mathrm{~min}$, as described ${ }^{26}$.

Cluster analysis and statistical tests. Custer analysis was performed based on $\triangle$ TBTN over STVE versus dark of individual neurons using an unsupervised agglomerative hierarchical tree method in MATLAB (linkage.m) based upon their pair-wise vectorial distance in the constructed 2D space (pdist.m) ${ }^{20}$.

All data are presented as mean \pm s.e.m. Data are considered significantly different when $p$ values are less than 0.05 . Where noted, either two-tailed Student's $t$ test or nonparametric Wilcoxon sign rank test was performed for within-cell comparison. For comparisons of multiple groups, either ANOVA with Newman-Keuls test or Kruskal-Wallis test with post hoc Mann-Whitney $U$ test were performed. The statistical test used for each experiment is specified in the results. Sample size for each experiment was confirmed with power test using the program $G^{*}$ power.

Experiments and analysis were performed blind to the experimental conditions.

Data availability. The data sets generated during and/or analyzed during the current study are available from the corresponding authors.

Received: 9 January 2018 Accepted: 8 June 2018

Published online: 24 July 2018

\section{References}

1. Froemke, R. C. Plasticity of cortical excitatory-inhibitory balance. Annu. Rev. Neurosci. 38, 195-219 (2015).

2. Hartman, K. N., Pal, S. K., Burrone, J. \& Murthy, V. N. Activity-dependent regulation of inhibitory synaptic transmission in hippocampal neurons. Nat. Neurosci. 9, 642-649 (2006).

3. Huang, Z. J., Di Cristo, G. \& Ango, F. Development of GABA innervation in the cerebral and cerebellar cortices. Nat. Rev. Neurosci. 8, 673-686 (2007).

4. Jiang, B., Huang, Z. J., Morales, B. \& Kirkwood, A. Maturation of GABAergic transmission and the timing of plasticity in visual cortex. Brain Res. Brain Res. Rev. 50, 126-133 (2005).

5. Liu, Y., Zhang, L. I. \& Tao, H. W. Heterosynaptic scaling of developing GABAergic synapses: dependence on glutamatergic input and developmental stage. J. Neurosci. 27, 5301-5312 (2007).

6. Pieraut, S. et al. Experience-dependent remodeling of basket cell networks in the dentate gyrus. Neuron 84, 107-122 (2014).

7. Wamsley, B. \& Fishell, G. Genetic and activity-dependent mechanisms underlying interneuron diversity. Nat. Rev. Neurosci. 18, 299-309 (2017).

8. Akgul, G. \& McBain, C. J. Diverse roles for ionotropic glutamate receptors on inhibitory interneurons in developing and adult brain. J. Physiol. 594, 5471-5490 (2016).

9. Bredt, D. S. \& Nicoll, R. A. AMPA receptor trafficking at excitatory synapses. Neuron 40, 361-379 (2003).

10. Kessels, H. W. \& Malinow, R. Synaptic AMPA receptor plasticity and behavior. Neuron 61, 340-350 (2009).

11. Wu, G., Malinow, R. \& Cline, H. T. Maturation of a central glutamatergic synapse. Science 274, 972-976 (1996)

12. Zhou, Z. et al. The C-terminal tails of endogenous GluA1 and GluA2 differentially contribute to hippocampal synaptic plasticity and learning. Nat. Neurosci. 21, 50-62 (2018)
13. Shi, S., Hayashi, Y., Esteban, J. A. \& Malinow, R. Subunit-specific rules governing AMPA receptor trafficking to synapses in hippocampal pyramidal neurons. Cell 105, 331-343 (2001).

14. Haas, K., Li, J. \& Cline, H. T. AMPA receptors regulate experience-dependent dendritic arbor growth in vivo. Proc. Natl Acad. Sci. USA 103, 12127-12131 (2006).

15. Yoon, B. J., Smith, G. B., Heynen, A. J., Neve, R. L. \& Bear, M. F. Essential role for a long-term depression mechanism in ocular dominance plasticity. Proc. Natl Acad. Sci. USA 106, 9860-9865 (2009).

16. Shen, W., McKeown, C. R., Demas, J. A. \& Cline, H. T. Inhibition to excitation ratio regulates visual system responses and behavior in vivo. J. Neurophysiol. 106, 2285-2302 (2011).

17. Rumpel, S., LeDoux, J., Zador, A. \& Malinow, R. Postsynaptic receptor trafficking underlying a form of associative learning. Science 308, 83-88 (2005).

18. Akerman, C. J. \& Cline, H. T. Refining the roles of GABAergic signaling during neural circuit formation. Trends Neurosci. 30, 382-389 (2007).

19. Khakhalin, A. S., Koren, D., Gu, J., Xu, H. \& Aizenman, C. D. Excitation and inhibition in recurrent networks mediate collision avoidance in Xenopus tadpoles. Eur. J. Neurosci. 40, 2948-2962 (2014).

20. He, H. Y., Shen, W., Hiramoto, M. \& Cline, H. T. Experience-dependent bimodal plasticity of inhibitory neurons in early development. Neuron $\mathbf{9 0}$, 1203-1214 (2016).

21. Aizenman, C. D., Munoz-Elias, G. \& Cline, H. T. Visually driven modulation of glutamatergic synaptic transmission is mediated by the regulation of intracellular polyamines. Neuron 34, 623-634 (2002).

22. Chen, Y. et al. Activity-induced Nr4al regulates spine density and distribution pattern of excitatory synapses in pyramidal neurons. Neuron 83, 431-443 (2014).

23. Gambrill, A. C., Faulkner, R. \& Cline, H. T. Experience-dependent plasticity of excitatory and inhibitory intertectal inputs in Xenopus tadpoles. J. Neurophysiol. 00611, 02016 (2016).

24. Richards, B. A., Voss, O. P. \& Akerman, C. J. GABAergic circuits control stimulus-instructed receptive field development in the optic tectum. Nat. Neurosci. 13, 1098-1106 (2010).

25. Tao, H. W. \& Poo, M. M. Activity-dependent matching of excitatory and inhibitory inputs during refinement of visual receptive fields. Neuron $\mathbf{4 5}$, 829-836 (2005)

26. Shen, W. et al. Acute synthesis of CPEB is required for plasticity of visual avoidance behavior in Xenopus. Cell Rep. 6, 737-747 (2014).

27. Volk, L., Chiu, S. L., Sharma, K. \& Huganir, R. L. Glutamate synapses in human cognitive disorders. Annu. Rev. Neurosci. 38, 127-149 (2015).

28. Rapanelli, M., Frick, L. R. \& Pittenger, C. The role of interneurons in autism and tourette syndrome. Trends Neurosci. 40, 397-407 (2017).

29. Fuchs, E. C. et al. Recruitment of parvalbumin-positive interneurons determines hippocampal function and associated behavior. Neuron 53, 591-604 (2007).

30. Gu, Y. et al. Obligatory role for the immediate early gene NARP in critical period plasticity. Neuron 79, 335-346 (2013).

31. Pelkey, K. A. et al. Pentraxins coordinate excitatory synapse maturation and circuit integration of parvalbumin interneurons. Neuron 85, 1257-1272 (2015).

32. Gainey, M. A., Hurvitz-Wolff, J. R., Lambo, M. E. \& Turrigiano, G. G. Synaptic scaling requires the GluR2 subunit of the AMPA receptor. J. Neurosci. 29, 6479-6489 (2009).

33. Cohen, S. M. et al. Excitation-transcription coupling in parvalbumin-positive interneurons employs a novel CaM Kinase-dependent pathway distinct from excitatory neurons. Neuron 90, 292-307 (2016).

34. Geiger, J. R. et al. Relative abundance of subunit mRNAs determines gating and $\mathrm{Ca}^{2+}$ permeability of AMPA receptors in principal neurons and interneurons in rat CNS. Neuron 15, 193-204 (1995).

35. Chen, J. L. et al. Clustered dynamics of inhibitory synapses and dendritic spines in the adult neocortex. Neuron 74, 361-373 (2012).

36. Xue, M., Atallah, B. V. \& Scanziani, M. Equalizing excitation-inhibition ratios across visual cortical neurons. Nature 511, 596-600 (2014).

37. Joseph, A. \& Turrigiano, G. G. All for one but not one for all: excitatory synaptic scaling and intrinsic excitability are coregulated by CaMKIV, whereas inhibitory synaptic scaling is under independent control. J. Neurosci. 37, 6778-6785 (2017).

38. Lu, W., Bushong, E. A., Shih, T. P., Ellisman, M. H. \& Nicoll, R. A. The cell-autonomous role of excitatory synaptic transmission in the regulation of neuronal structure and function. Neuron 78, 433-439 (2013).

39. Peng, Y. R. et al. Postsynaptic spiking homeostatically induces cellautonomous regulation of inhibitory inputs via retrograde signaling. $J$. Neurosci. 30, 16220-16231 (2010).

40. Anderson, G. R. et al. Candidate autism gene screen identifies critical role for cell-adhesion molecule CASPR2 in dendritic arborization and spine development. Proc. Natl Acad. Sci. USA 109, 18120-18125 (2012). 
41. Varea, O. et al. Synaptic abnormalities and cytoplasmic glutamate receptor aggregates in contactin associated protein-like 2/Caspr2 knockout neurons. Proc. Natl Acad. Sci. USA 112, 6176-6181 (2015).

42. Fritschy, J. M. Epilepsy E/I balance and GABA(A) receptor plasticity. Front. Mol. Neurosci. 1, 5 (2008).

43. Lewis, D. A., Hashimoto, T. \& Volk, D. W. Cortical inhibitory neurons and schizophrenia. Nat. Rev. Neurosci. 6, 312-324 (2005).

44. Nelson, S. B. \& Valakh, V. Excitatory/inhibitory balance and circuit homeostasis in autism spectrum disorders. Neuron 87, 684-698 (2015)

45. Banerjee, A. et al. Jointly reduced inhibition and excitation underlies circuitwide changes in cortical processing in Rett syndrome. Proc. Natl Acad. Sci. USA 113, E7287-E7296 (2016).

46. Lu, B., Wang, K. H. \& Nose, A. Molecular mechanisms underlying neural circuit formation. Curr. Opin. Neurobiol. 19, 162-167 (2009).

47. Nugent, F. S., Penick, E. C. \& Kauer, J. A. Opioids block long-term potentiation of inhibitory synapses. Nature 446, 1086-1090 (2007).

48. Bloodgood, B. L. \& Sabatini, B. L. $\mathrm{Ca}^{2+}$ signaling in dendritic spines. Curr. Opin. Neurobiol. 17, 345-351 (2007).

49. Gu, X., Zhou, L. \& Lu, W. An NMDA receptor-dependent mechanism underlies inhibitory synapse development. Cell Rep. 14, 471-478 (2016).

50. Danielson, E. \& Lee, S. H. SynPAnal: software for rapid quantification of the density and intensity of protein puncta from fluorescence microscopy images of neurons. PLoS ONE 9, el15298 (2014).

\section{Acknowledgements}

We thank Dr. Richard Huganir for sharing the GluAl antibody and Dr. Eric Danielson for providing the SynPAnal puncta analysis software. We thank Dr. Daniel Choquet, Dr. Anton Maximov, Dr. Richard Huganir and members of the Cline lab for critical discussions. This work was supported by the NEI (EY011261 and EY027437) and the Hahn Family Foundation to H.T.C., and National Nature Sciences Foundation of China (NSFC 31271176) and Zhejiang Provincial Natural Science Foundation of China (LY17C090007) to W.S.

\section{Author contributions}

Designed research: H.-y.H., W.S. and H.T.C., Performed research: H.-y.H., W.S., L.Z. and X.G., Contributed analytic tools: H.-y.H. and W.S., Analyzed data: H.-y.H., W.S. and H.T.C., Wrote the paper: H.-y.H., W.S. and H.T.C.

\section{Additional information}

Supplementary Information accompanies this paper at https://doi.org/10.1038/s41467018-05125-4.

Competing interests: The authors declare no competing interests.

Reprints and permission information is available online at http://npg.nature.com/ reprintsandpermissions/

Publisher's note: Springer Nature remains neutral with regard to jurisdictional claims in published maps and institutional affiliations.

(c) (i) Open Access This article is licensed under a Creative Commons Attribution 4.0 International License, which permits use, sharing, adaptation, distribution and reproduction in any medium or format, as long as you give appropriate credit to the original author(s) and the source, provide a link to the Creative Commons license, and indicate if changes were made. The images or other third party material in this article are included in the article's Creative Commons license, unless indicated otherwise in a credit line to the material. If material is not included in the article's Creative Commons license and your intended use is not permitted by statutory regulation or exceeds the permitted use, you will need to obtain permission directly from the copyright holder. To view a copy of this license, visit http://creativecommons.org/ licenses/by/4.0/.

(C) The Author(s) 2018 